\title{
The Yarkovsky Drift's Influence on NEAs: Trends and Predictions with NEOWISE Measurements
}

\author{
C. R. Nugent ${ }^{1}$, A. Mainzer ${ }^{2}$, J. Masiero ${ }^{2}$, T. Grav $^{3}$, and J. Bauer ${ }^{2}$
}

Received —

\footnotetext{
${ }^{1}$ Department of Earth and Space Sciences, University of California, Los Angeles, CA 90095, USA

${ }^{2}$ Jet Propulsion Laboratory, California Institute of Technology, Pasadena, CA 91109 USA

${ }^{3}$ Planetary Science Institute, Tucson, AZ
} 


\begin{abstract}
We used WISE-derived geometric albedos $\left(p_{V}\right)$ and diameters, as well as geometric albedos and diameters from the literature, to produce more accurate diurnal Yarkovsky drift predictions for 540 near-Earth asteroids (NEAs) out of the current sample of $\sim 8800$ known objects. As ten of the twelve objects with the fastest predicted rates have observed arcs of less than a decade, we list upcoming apparitions of these NEAs to facilitate observations.
\end{abstract}

Subject headings: astrometry — minor planets, asteroids — minor planets, asteroids: individual (2010 JG87, 2006 HY51, (137924) 2000 BD19, 2010 HX107, 2002 LT24, (153201) 2000 WO107, 2010 EX11, 2008 EY5, 2006 NL, 2006 MD12, 2010 GQ75) radiation mechanisms: thermal 


\section{Introduction}

The Yarkovsky effect is a non-gravitational force that perturbs the orbits of small bodies, including near-Earth asteroids (NEAs). Despite its small magnitude, it must be

included in the calculation of precise asteroid trajectory predictions (Giorgini et al. 2002; Milani et al. 2009), and it is believed to be a key mechanism in the process that delivers asteroids from the main belt to near-Earth space (Bottke et al. 2006).

The diurnal Yarkovsky effect (or drift) is caused by anisotropic re-radiation of absorbed sunlight. It is driven by the thermal properties of an asteroid as well as the amount of absorbed incident radiation. A given surface point on an asteroid observes maximum incident radiation at local noon, but thermal inertia causes the time of maximum emitted radiation (usually at infrared wavelengths) to occur later. Each arriving and departing photon has an associated momentum $p=E / c$, where $E$ is the photon's energy and $c$ is the speed of light. Since the body is rotating, the incident radiation is in a different direction than the later emitted radiation, and the body experiences a very small net acceleration. If the body has a prograde spin, the net acceleration has a component aligned with the motion of the body's orbit, nudging the body away from the sun. Similarly, a body with a retrograde spin will feel an acceleration with a component anti-aligned with its velocity, shifting it towards the sun (Bottke et al. 2006).

There is also a seasonal component to the Yarkovsky effect. The seasonal Yarkovsky effect is largest when an asteroid's obliquity is $90^{\circ}$, and goes to zero as obliquity approaches $0^{\circ}$ or $180^{\circ}$ (Bottke et al. 2006). Vokrouhlický et al. (2000) calculated the diurnal and seasonal components of the Yarkovsky effect for several objects, and in all cases the seasonal component was significantly smaller. Even in the case of (1566) Icarus, which has an obliquity equal to $103^{\circ}$, the diurnal component for this object was more than twice the magnitude of the seasonal component over a range of likely thermal conductivities. 
There have been few direct measurements of the Yarkovsky drift. Chesley et al. (2003) used radar ranging to make the first direct detection of the Yarkovsky drift. They measured the rate of change of (6489) Golevka's semi-major axis $(d a / d t)$ to be of order $10^{-4} \mathrm{AU} / \mathrm{Myr}$. A magnitude $d a / d t$ of $10^{-3}$ AU/Myr Yarkovsky drift was associated with asteroid 1992 BF by linking modern astrometry with observations from 1952 (Vokrouhlický et al. 2008). Nugent et al. (2012) used an orbit-fitting method to measure Yarkovsky drifts for 54 NEAs, and found an average rate magnitude of $(10.4 \pm 11.4) \times 10^{-4} \mathrm{AU} / \mathrm{Myr}$.

The Yarkovsky effect has been modeled by several researchers (Vokrouhlický et al. (2000); Spitale \& Greenberg (2001), for example). Mathematical formulations, such as those by Vokrouhlický et al. (2000), indicate that Yarkovsky drift is inversely proportional to diameter. Although the amount of absorbed radiation increases with the square of the diameter, mass increases with the cube of the diameter $(D)$, so drift rate is expected to show a $1 / D$ dependence.

However, because thermal inertia could also depend on size, the size-dependence of Yarkovsky drift could be more complicated than presently assumed. Theory predicts that the more massive a body is, the more regolith it should retain (Scheeres et al. 2002), and regolith may act as an insulating blanket (though for bodies smaller than $10 \mathrm{~km}$ in diameter, spin state may be more indicative of regolith presence). Low porosity and high thermal inertia should create a longer time lag between absorbed radiation and thermal re-radiation, perhaps resulting in a stronger Yarkovsky effect (depending on the rotation state).

Additionally, these models incorporate physical properties of asteroids that are often poorly measured. Although obliquity, heat capacity, thermal conductivity, and bulk density are generally difficult to quantify, more basic properties such as geometric albedo $\left(p_{V}\right)$ and diameter can be ill-constrained. This dearth of information has hindered the accuracy of 
Yarkovsky predictions.

The Wide-field Infrared Survey Explorer (WISE) (Wright et al. 2010) has observed over 150,000 minor planets (including 600 NEAS) at infrared wavelengths (Mainzer et al. 2011a). It is these infrared measurements, combined with optical observations, that can separate the contributions of size and $p_{V}$ to the observed flux. The dependence of flux on $p_{V}$ is weaker in the thermal wavelengths, since the majority of the light emitted from the asteroid is from thermal emission, not reflected infrared sunlight. With a thermal model that incorporates both infrared and optical observations, size and $p_{V}$ can be determined. With thermally-dominated WISE wavelengths, it has been shown that for asteroids observed with good signal-to-noise ratios and relatively low amplitude lightcurve variations, diameter can be determined to within $\pm 10 \%$, and $p_{V}$ can be determined to within $\pm 25 \%$ of the amount of the albedo (Mainzer et al. 2011c,d). Combining WISE measurements with published reliable diameters determined from in situ spacecraft visits, stellar occultations, and radar produces a list of NEOs with well-determined diameters and geometric albedos.

\section{Methods}

We employed the mathematical formulation of the diurnal Yarkovsky effect developed by Vokrouhlický et al. (2000) to numerically estimate Yarkovsky drifts. Although our methods are not identical, this work follows that of Vokrouhlický et al. (2005), who predicted drifts for 28 NEAs. We expand from that foundation, incorporating newly available physical properties.

For a time step along an NEA's orbit, the Yarkovsky acceleration was computed following equation (1) of Vokrouhlický et al. (2000). This equation assumes a spherical body and that temperatures throughout the body do not greatly deviate from an average 
temperature. Obliquity was assumed to be $0^{\circ}$ to produce maximum drift. Therefore, the reported drifts in this paper are upper limits. Additionally, a $0^{\circ}$ obliquity assumes that all drift is due to the diurnal Yarkovsky effect, as the seasonal Yarkovsky effect has zero magnitude for this case (Bottke et al. 2006). This acceleration was resolved along orthogonal directions, and Gauss' form of Lagrange's planetary equations (Danby 1992) was employed to evaluate an orbit-averaged $d a / d t$.

The magnitude of the diurnal Yarkovsky drift depends on physical parameters which can be ill-defined. The drift magnitude is not linearly related to these unknown parameters, and so the resultant drift magnitude was statistically modeled to more accurately determine the effect of these uncertainties. For each NEA, we used $p_{V}$ and diameter measurements derived by WISE (Mainzer et al. 2011b) or other sources of reliable diameter and $p_{V}$ measurements in the literature, primarily radar detections and stellar occultations. We employed a Monte Carlo method to explore how variations in physical parameters contribute to errors in the prediction of $d a / d t$. For 1000 realizations per NEA, we added Gaussian-distributed noise to the diameter and $p_{V}$ measurements, so that standard distribution of the noise corresponded to the $1 \sigma$ error bars on those measurements.

As the formulation of Vokrouhlický et al. (2000) relies on Bond albedo $A$, we approximated A using $A \approx(0.290+0.684 G) p_{V}$, where $G$ is the slope parameter (Bowell et al. 1989). In seven cases, $G$ was available in the JPL Small-Bodies database (Chamberlin 2008). In the remaining cases, $G$ was taken to equal 0.15, as this was the value used to compute physical properties of NEAs in Mainzer et al. (2011a), based on the standard value assumed by the Minor Planet Center for computing $H$.

Additionally, we varied the thermal conductivity, bulk density, and density of the surface layer between the ranges shown in Table 1. The physical parameters in Table 1 were chosen to represent a range of asteroid compositions, so that our Yarkovsky estimates 
would represent reasonable estimates of the range of physical properties of rocky asteroids. At one end of the spectrum are physical parameters mimicking a low-density rubble pile, at the other, a regolith-free rock chunk.

Emissivity was always assumed to be 0.9 . If rotation rate was not available in the JPL Database (Chamberlin 2008), the rotation rate was assumed to be 5 revolutions/day, based on the average spin rate values for asteroids 1 to $10 \mathrm{~km}$ in diameter shown in Figure 1 of Pravec \& Harris (2000). Rotation rates were unavailable for $81 \%$ of the NEAs.

The $d a / d t$ values quoted in this paper are the mean of these 1000 realizations. Error bars on $d a / d t$ were determined by computing the standard deviation from the mean.

Table 1: Physical and thermal properties used for generating predictions of $d a / d t$ drifts. Thermal properties are based on the work of Opeil et al. (2010), who measured three meteorites at $200 \mathrm{~K}$. Listed are heat capacity $C$, thermal conductivity $K$, bulk density of the surface $\rho_{s}$, and mean bulk density $\rho_{b}$. The surface and bulk densities are assumed to have a similar range of values, however, $\rho_{s}$ was not necessarily equal to $\rho_{b}$ for a given object and realization.

\begin{tabular}{lccc}
\hline \hline Composition & $C\left(\mathrm{~J} \mathrm{~kg}^{-1} \mathrm{~K}^{-1}\right)$ & $K\left(\mathrm{~W} \mathrm{~m}^{-1} \mathrm{~K}^{-1}\right)$ & $\left.\rho_{s} / \rho_{b}\left(\mathrm{~kg} \mathrm{~m}^{-3}\right)\right)$ \\
\hline Rubble Pile & 500 & 0.01 & 1000 \\
Rock Chunk & 500 & 0.50 & 3000 \\
\hline
\end{tabular}

\section{Results}

We estimated diurnal Yarkovsky drifts for 540 NEAs with measured diameters and geometric albedos. The dozen objects with the highest drifts are listed in Table 2, upcoming apparitions of those objects are in Table 3, and predicted drifts for all objects are in 
Table 4. Tables 2 and 4 include an order of magnitude estimate of along-track displacement $(\Delta \rho)$ that would result from the $d a / d t$ drift over 10 years. For this we use the following formulation from Vokrouhlický et al. (2000),

$$
\Delta \rho \simeq 7 \dot{a}_{4}\left(\Delta_{10} t\right)^{2} a_{A U}^{-3 / 2}
$$

where $\Delta \rho$ is in units of $\mathrm{km}, \dot{a}_{4}$ is $d a / d t$ in units of $10^{-4} \mathrm{AU} / \mathrm{Myr}, \Delta_{10} t$ is the time difference between observations in tens of years, and $a_{A U}$ is the semimajor axis of the object in AU. We note that the four of the twelve objects with the largest predicted drifts were discovered by the NEOWISE portion of the WISE mission (2010 JG87, 2010 HX107, 2010 EX11, and 2010 GQ75).

Individual realizations for the NEA with the fastest predicted drift, $2010 \mathrm{JG} 87$, are examined in Figures 1 and 2. In each of these figures, all 1000 realizations of physical parameter combinations are shown, so their individual influences are apparent for this object.

2010 JG87's diameter was determined to within $\pm 10 \%$ (Mainzer et al. 2011b) based on the WISE observations (Figure 1), and as diameter and bulk density are used to estimate mass, it is the uncertainty in bulk density that mainly determines the predicted drift for this object (Figure 2). Surface density and thermal conductivity both contribute to the thermal lag, and for this object, low values of $K$ and $\rho_{s}$ lead to a thermal lag that produces the strongest drifts (given the object's assumed rotation period of 5 revolutions/day). As geometric albedo has been determined to be $0.20 \pm 0.04$ for this object, the range of geometric albedo values explored do not strongly influence the resulting drift.

We now examine the values that govern Yarkovsky strength for all objects in our sample. As we are comparing the mean $d a / d t$ values of each object, the following compares drifts effectively computed with the same bulk density and density of the surface layer. The predicted diurnal $d a / d t$ has a 1/D dependence, and also depends on the amount of average 
incident radiation the NEA receives per orbit and $d a / d t$.

The 1/D dependence can be seen in Figure 3. As all objects in these plots are assumed to have the same bulk density $\left(2000 \mathrm{~kg} \mathrm{~m}^{-3}\right)$, it is only the difference in diameters that produces different mass estimates.

After diameter, the second parameter that strongly influences drift magnitude is the average incident radiation per orbit, as seen in Figure 3. The more light received by the NEA over its orbit, the more light is available for re-emission and the loss of momentum that powers the drift.

Many values of $d a / d t$ reported in Table 4 have large error bars due to uncertainties in physical properties. Observations that further constrain the obliquity, density, rotation rate, thermal conductivity and heat capacity would also constrain predicted drift rates. Measurements of thermal properties of these objects would be valuable, as would the measurement of rotation rates and obliquities (either from lightcurves or radar observations). It's expected that $1 / 6$ objects larger than 200 meters are binary systems (Margot et al. 2002; Pravec et al. 2006), a property which could enable density measurements.

Historically, Yarkovsky detections require either radar observations over three apparitions (Chesley et al. 2003) or optical observations that meet a set of criteria. Nugent et al. (2012) required an object to (1) have an observed arc of at least $\sim 15$ years, (2) have observations distributed throughout that arc in time (defined as at least 8 observations per orbit for at least 5 orbits) and (3) have a fraction of these observations at favorable geometries and distances (defined by the Yarkovsky sensitivity $s_{Y}>2.0$ ).

None of the objects in Table 2 have enough optical or radar observations to meet the above criteria for detection. Therefore, when possible we encourage the community to observe these objects and contribute astrometry to the Minor Planet Center. More 


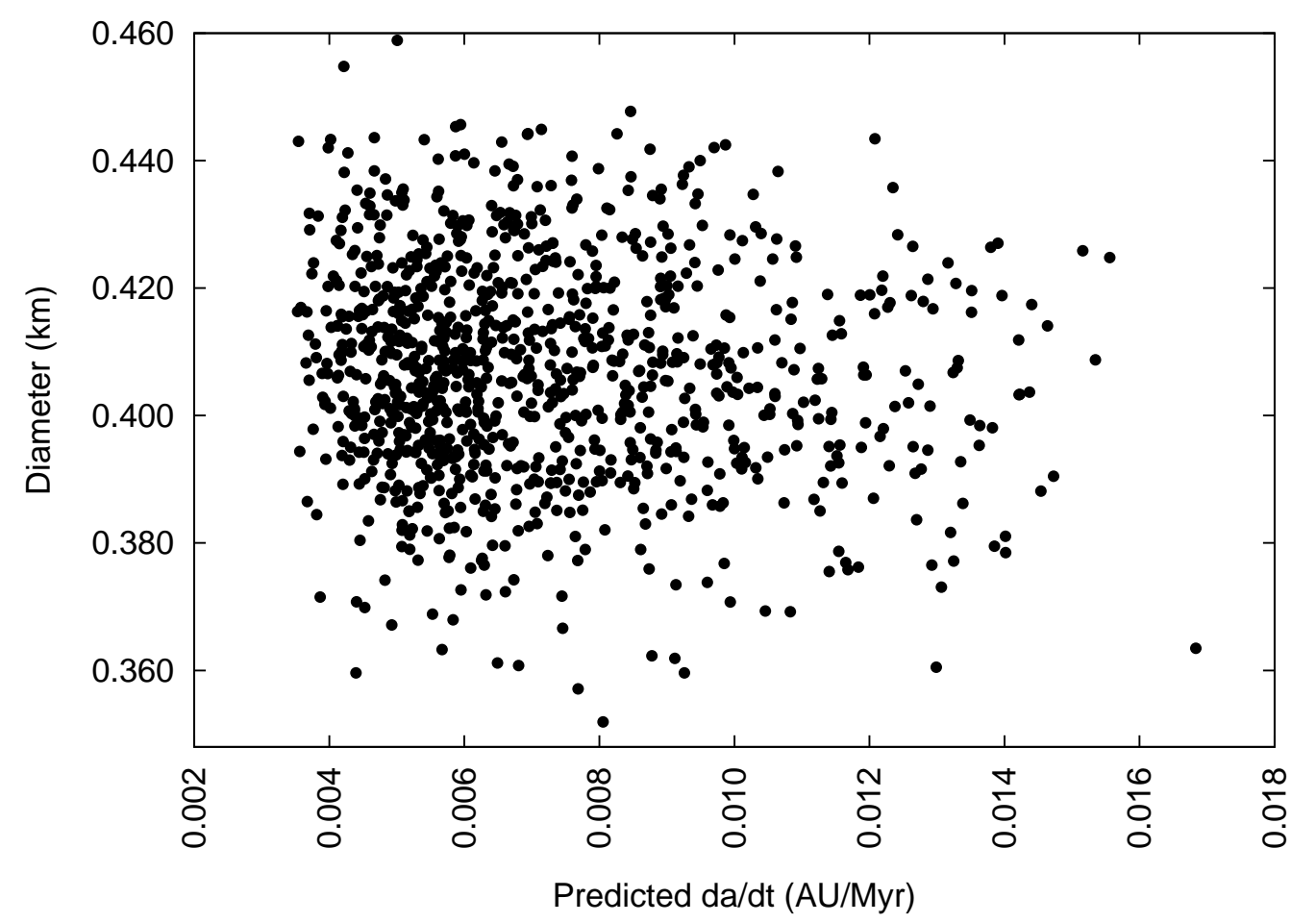

Fig. 1.- 1000 realizations of diameter vs $d a / d t$ for NEA 2010 JG87. Each point represents the drift produced by a different combination of physical parameters. This object has the fastest predicted diurnal drift of all the NEAs in this paper, with $d a / d t=(72.11 \pm 25.12) \times$ $10^{-4} \mathrm{AU} / \mathrm{Myr}$. Although Yarkovsky drift has a $1 / D$ dependence, the relatively small error bars on this object's diameter (and therefore the small range of diameters shown in this plot), combined with the variations in the other parameters (surface density, bulk density, thermal conductivity $K, p_{v}$, and $G$ ) prevent this dependence from being immediately apparent in this figure. For a clearer illustration of the relationship between $d a / d t$ and diameter, see Figure 3. For the relationship between $d a / d t$ and the other physical properties that were varied during each realization, see Figure 2 . 

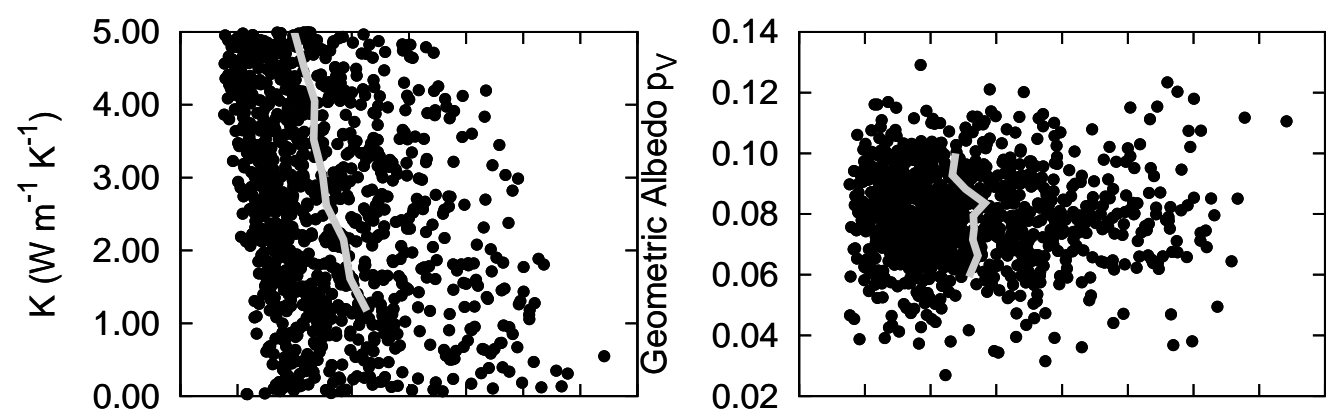

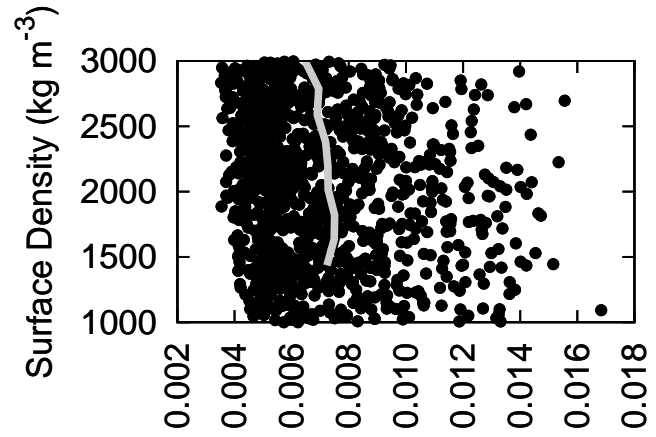

Predicted da/dt (AU/Myr)

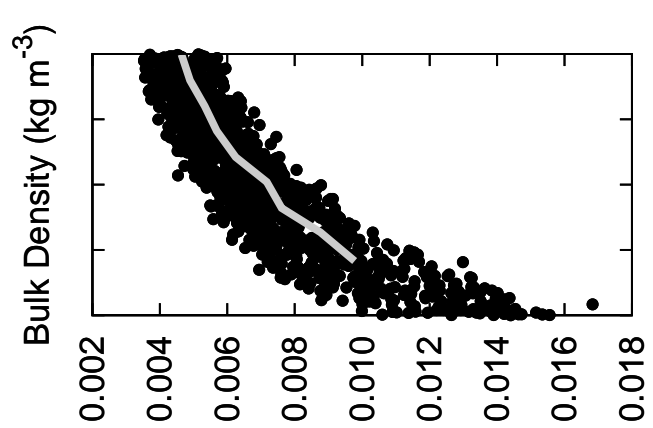

Predicted da/dt (AU/Myr)

Fig. 2.- 1000 realizations of predicted diurnal $d a / d t$ drift for 2010 JG87, the NEA with the fastest predicted diurnal drift in this paper. For each realization, diameter, thermal conductivity, geometric albedo, slope parameter $G$, density of the surface layer, and bulk density were varied as described in the text. Grey lines are running averages. For this object, it is the uncertainty in bulk density that is mainly responsible for the span of calculated $d a / d t$ drifts, as the diameter of this object is well-constrained (see Figure 1). Also visible are the relationships between thermal conductivity and surface density and drift. These two properties govern the thermal lag angle. 


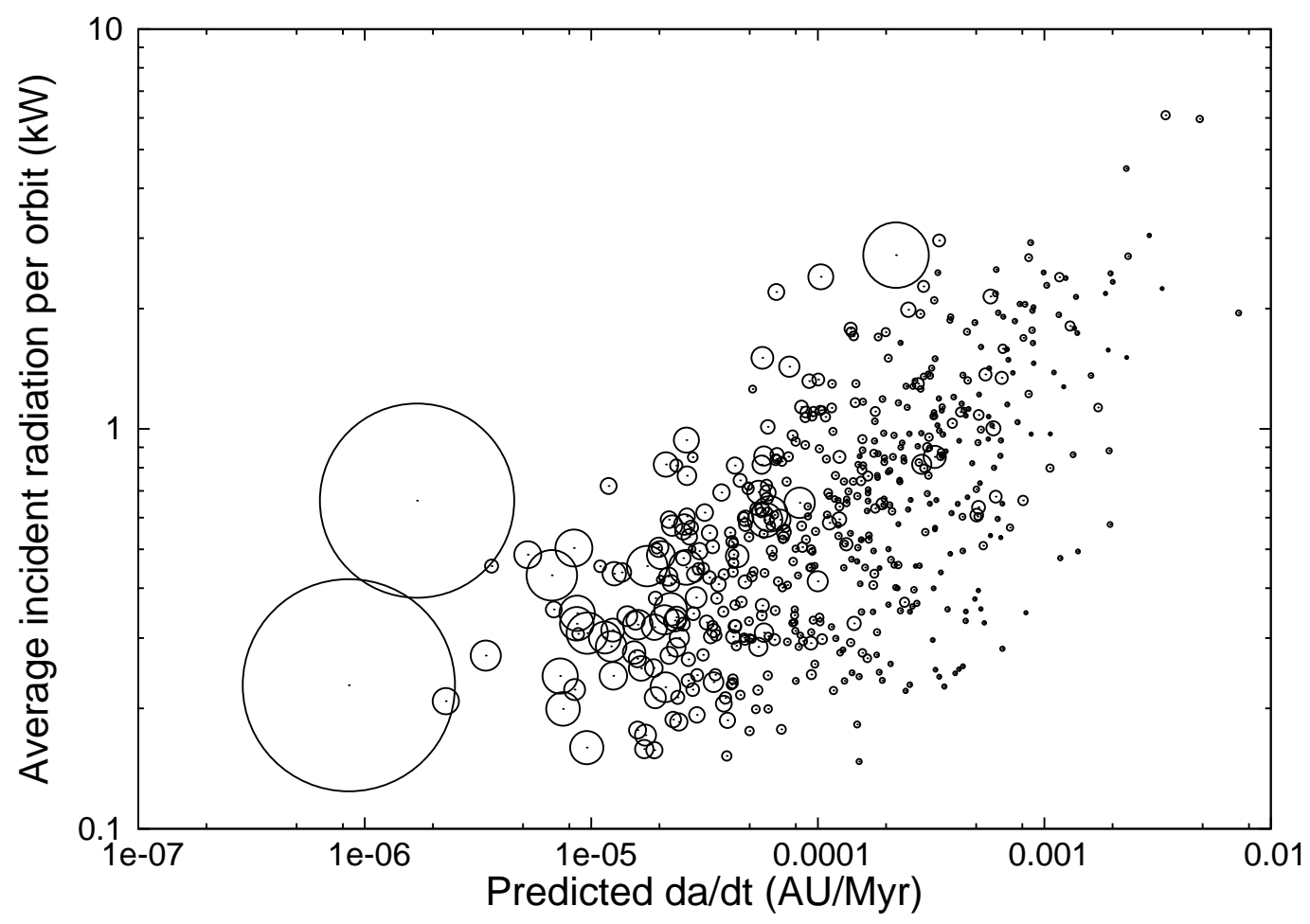

Fig. 3.- Relationship between the average incident radiation each NEA receives per orbit and predicted diurnal $d a / d t$ drift. Circle sizes are proportional to the diameter of the object. The more sunlight an object receives during its orbit, the more power is available to the Yarkovsky drift. However, this link is tempered by the the diameter- larger objects experience a smaller drift than smaller objects, given the same average incident radiation. 
astrometry is needed for all these objects to enable a future Yarkovsky detection via a fit to optical-only data.

To facilitate these observations, Table 3 provides apparitions and associated apparent magnitude ranges for these objects between April 1st, 2012 and April 1st, 2022. These apparitions are defined as the times when the object's elongation is greater than $90^{\circ}$, and were generated using the JPL's Horizons ephemeris computation service.

The worldwide community of amateur and professional follow up observers is encouraged to consult this table when planning their observations. Several of the brighter objects may also be automatically picked up by sky surveys such as (in order of decreasing number of observations) PanSTARRS, the Catalina Sky Survey, Spacewatch, and the Lincoln Near Earth Asteroid Research Program (Larson 2007; McMillan 2007; Stokes et al. 2000). However, some of these objects only have brief windows where their elongation is greater than $90^{\circ}$ and $V<20.5 \mathrm{mag}$ (which is roughly the sensitivity limit of most surveys) and may be missed without special attention. The two brightest objects are likely to be automatically observed by surveys, however, additional observations that expanded coverage over the orbit in mean anomaly would be useful.

Unfortunately, not all objects are easily observable. 2010 EX11 does not have an elongation greater than $90^{\circ}$ during that time span, though on two apparitions (in 2012 and 2013) it does exceed $60^{\circ}$. Several of the remaining objects are extremely faint, with $V$ (mag) rarely brighter than 23.5. Although these observations may be challenging, they are vital for well-defined orbits and future Yarkovsky detections. 


\section{Conclusion}

In this paper we use WISE-derived geometric albedos and diameters, as well as values for geometric albedos and diameters published in the literature, to produce more accurate diurnal Yarkovsky drift predictions for 540 NEAs. Table 2 lists the 12 objects in our sample with the fastest rates, and Table 3 gives their apparitions over the next decade. Three of these objects have observed arcs of less than a year, and we encourage observers to obtain more astrometry of these objects when possible. Predicting which NEAs are most likely to be subject to strong Yarkovsky drifts relies upon robust determinations of asteroid physical properties, underscoring the need to continue to obtain such characterization data.

This publication makes use of data products from the Wide-field Infrared Survey Explorer, which is a joint project of the University of California, Los Angeles, and the Jet Propulsion Laboratory/California Institute of Technology, funded by the National Aeronautics and Space Administration. This publication also makes use of data products from NEOWISE, which is a project of the Jet Propulsion Laboratory/ California Institute of Technology, funded by the Planetary Science Division of the National Aeronautics and Space Administration. We gratefully acknowledge the extraordinary services specific to NEOWISE contributed by the International Astronomical Unions Minor Planet Center, operated by the Harvard-Smithsonian Center for Astrophysics, and the Central Bureau for Astronomical Telegrams, operated by Harvard University. We also thank the worldwide community of dedicated amateur and professional astronomers devoted to minor planet follow-up observations. This research has made use of the NASA/IPAC Infrared Science Archive, which is operated by the Jet Propulsion Laboratory, California Institute of Technology, under contract with the National Aeronautics and Space Administration.

We are very grateful for the work of David Vokrouhlický, particularly his 2000 paper, 
which is the foundation of this work. We would also wish to thank Jean-Luc Margot, whose comments markedly improved the manuscript. Finally, we also thank our referee for comments that materially improved the quality of this work. 


\section{REFERENCES}

Bottke, Jr., W. F., Vokrouhlický, D., Rubincam, D. P., \& Nesvorný, D. 2006, Annual Review of Earth and Planetary Sciences, 34, 157

Bowell, E., Hapke, B., Domingue, D., Lumme, K., Peltoniemi, J., \& Harris, A. W. 1989, in Asteroids II, 524-556

Chamberlin, A. B. 2008, Bulletin of the American Astronomical Society, 40

Chesley, S., Ostro, S., Vokrouhlicky, D., Capek, D., Giorgini, J., Nolan, M., Margot, J. L., Hine, A., Benner, L., \& Chamberlin, A. 2003, Science, 302, 1739

Danby, J. M. A. 1992, Fundamentals of Celestial Mechanics (Willmann-Bell, Inc)

Giorgini, J., Ostro, S., Benner, L., Chodas, P., Chesley, S., Hudson, R., Nolan, M., Klemola, A., Standish, E., Jurgens, R., Rose, R., Chamberlain, A., Yeomans, D., \& Margot, J. 2002, Science, 296, 132

Larson, S. 2007, in Near Earth Objects, our Celestial Neighbors: Opportunity and Risk, Proceedings if IAU Symposium 236, ed. A. M. G. B. Valsecchi, D. Vokrouhlický (Cambridge University Press), 323-328

Mainzer, A., Bauer, J., Grav, T., Masiero, J., Cutri, R. M., Dailey, J., Eisenhardt, P., McMillan, R. S., Wright, E., Walker, R., Jedicke, R., Spahr, T., Tholen, D., Alles, R., Beck, R., Brandenburg, H., Conrow, T., Evans, T., Fowler, J., Jarrett, T., Marsh, K., Masci, F., McCallon, H., Wheelock, S., Wittman, M., Wyatt, P., DeBaun, E., Elliott, G., Elsbury, D., Gautier, IV, T., Gomillion, S., Leisawitz, D., Maleszewski, C., Micheli, M., \& Wilkins, A. 2011a, The Astrophysical Journal, 731, 53

Mainzer, A., Grav, T., Bauer, J., Masiero, J., McMillan, R. S., Cutri, R. M., Walker, R., Wright, E., Eisenhardt, P., Tholen, D. J., Spahr, T., Jedicke, R., Denneau, L., 
DeBaun, E., Elsbury, D., Gautier, T., Gomillion, S., Hand, E., Mo, W., Watkins, J., Wilkins, A., Bryngelson, G. L., Del Pino Molina, A., Desai, S., Gómez Camus, M., Hidalgo, S. L., Konstantopoulos, I., Larsen, J. A., Maleszewski, C., Malkan, M. A., Mauduit, J.-C., Mullan, B. L., Olszewski, E. W., Pforr, J., Saro, A., Scotti, J. V., \& Wasserman, L. H. 2011b, The Astrophysical Journal, 743, 156

Mainzer, A., Grav, T., Masiero, J., Bauer, J., Wright, E., Cutri, R., McMillan, R. S., Cohen, M., Ressler, M., \& Eisenhardt, P. 2011c, The Astronomical Journal, 736

Mainzer, A., Grav, T., Masiero, J., Bauer, J., Wright, E., Cutri, R. M., Walker, R., \& McMillan, R. S. 2011d, The Astrophysical Journal Letters, 737, L9

Margot, J. L., Nolan, M. C., Benner, L. A. M., Ostro, S. J., Jurgens, R. F., Giorgini, J. D., Slade, M. A., \& Campbell, D. B. 2002, Science, 296, 1445

McMillan, R. S. 2007, in Near Earth Objects, our Celestial Neighbors: Opportunity and Risk, Proceedings if IAU Symposium 236, ed. A. M. G. B. Valsecchi, D. Vokrouhlický (Cambridge University Press), 329-340

Milani, A., Chesley, S. R., Sansaturio, M. E., Bernardi, F., Valsecchi, G. B., \& Arratia, O. 2009, Icarus, 203, 460

Nugent, C. R., Margot, J. L., Chesley, S. R., \& Vokrouhlický, D. 2012, accepted

Opeil, C., Consolmagno, G., \& Britt, D. 2010, Icarus, 208, 449

Pravec, P. \& Harris, A. 2000, Icarus, 148, 12

Pravec, P. et al. 2006, Icarus, 181, 63

Scheeres, D. J., Durda, D. D., \& Geissler, P. E. 2002, Asteroids III, 527

Spitale, J. \& Greenberg, R. 2001, Icarus, 149, 222 
Stokes, G. H., Evans, J. B., Viggh, H. E. M., Shelly, F. C., \& Pearce, E. C. 2000, Icarus, 148,21

Vokrouhlický, D., Capek, D., Chesley, S., \& Ostro, S. 2005, Icarus, 173, 166

Vokrouhlický, D., Chesley, S. R., \& Matson, R. D. 2008, Astronomical Journal, 135, 2336

Vokrouhlický, D., Milani, A., \& Chesley, S. R. 2000, Icarus, 148, 118

Wright, E. L., Eisenhardt, P. R. M., Mainzer, A. K., Ressler, M. E., Cutri, R. M., Jarrett, T., Kirkpatrick, J. D., Padgett, D., McMillan, R. S., Skrutskie, M., Stanford, S. A., Cohen, M., Walker, R. G., Mather, J. C., Leisawitz, D., Gautier, III, T. N., McLean, I., Benford, D., Lonsdale, C. J., Blain, A., Mendez, B., Irace, W. R., Duval, V., Liu, F., Royer, D., Heinrichsen, I., Howard, J., Shannon, M., Kendall, M., Walsh, A. L., Larsen, M., Cardon, J. G., Schick, S., Schwalm, M., Abid, M., Fabinsky, B., Naes, L., \& Tsai, C.-W. 2010, The Astronomical Journal, 140, 1868

This manuscript was prepared with the AAS LATEX macros v5.2. 
E1

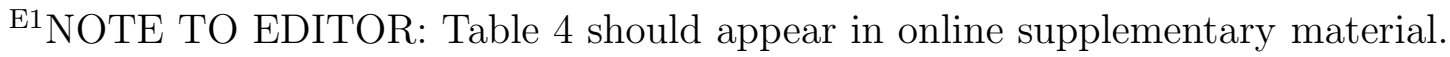


Table 2. The 12 NEAs with largest predicted Yarkovsky drift rates.

\begin{tabular}{|c|c|c|c|c|c|c|c|c|}
\hline NEA & $\begin{array}{c}a \\
(\mathrm{AU})\end{array}$ & $e$ & $\begin{array}{c}i \\
(\operatorname{deg})\end{array}$ & $\begin{array}{l}D \\
\quad(\mathrm{~km})\end{array}$ & $p_{V}$ & Arc & $\begin{array}{c}d a / d t \\
10^{-4} \mathrm{AU} / \mathrm{Myr}\end{array}$ & $\begin{array}{l}\Delta \rho \\
(\mathrm{km})\end{array}$ \\
\hline $2010 \mathrm{JG} 87$ & 2.76 & 0.95 & 16.91 & $0.41 \pm 0.02$ & $0.20 \pm 0.04$ & 54 days & $72.11 \pm 25.12$ & 110.0 \\
\hline 2006 HY 51 & 2.60 & 0.97 & 30.58 & $1.22 \pm 0.27$ & $0.16 \pm 0.09$ & 2006-2011 & $54.35 \pm 23.85$ & 90.8 \\
\hline 2007 EP88 & 0.84 & 0.89 & 20.78 & $0.64 \pm 0.04$ & $0.17 \pm 0.04$ & $2007-2010$ & $48.57 \pm 16.22$ & 443.8 \\
\hline (137924) 2000 BD19 & 0.88 & 0.89 & 25.69 & $0.97 \pm 0.04$ & $0.25 \pm 0.05$ & $1997-2010$ & $34.30 \pm 12.02$ & 292.6 \\
\hline 2010 HX107 & 0.80 & 0.30 & 3.37 & $0.06 \pm 0.01$ & $0.19 \pm 0.07$ & 60 days & $33.11 \pm 23.05$ & 323.6 \\
\hline 2002 LT24 & 0.72 & 0.50 & 0.76 & $0.14 \pm 0.02$ & $0.14 \pm 0.07$ & $2002-2010$ & $29.06 \pm 16.58$ & 333.1 \\
\hline (153201) 2000 WO107 & 0.91 & 0.78 & 7.78 & $0.51 \pm 0.08$ & $0.13 \pm 0.06$ & $2000-2010$ & $23.45 \pm 10.14$ & 188.7 \\
\hline 2010 EX11 & 0.96 & 0.11 & 9.75 & $0.04 \pm 0.01$ & $0.23 \pm 0.09$ & 37 days & $23.09 \pm 18.64$ & 173.0 \\
\hline 2008 EY5 & 0.63 & 0.63 & 5.07 & $0.36 \pm 0.01$ & $0.12 \pm 0.03$ & $2008-2011$ & $23.00 \pm 9.75$ & 324.6 \\
\hline $2006 \mathrm{NL}$ & 0.85 & 0.58 & 20.08 & $0.22 \pm 0.05$ & $0.46 \pm 0.14$ & 2006-2009 & $20.05 \pm 12.66$ & 179.8 \\
\hline 2006 MD12 & 0.84 & 0.61 & 27.27 & $0.27 \pm 0.06$ & $0.43 \pm 0.13$ & 2006-2009 & $19.60 \pm 12.15$ & 178.6 \\
\hline 2010 GQ75 & 2.43 & 0.87 & 43.23 & $0.37 \pm 0.02$ & $0.11 \pm 0.03$ & 34 days & $19.48 \pm 9.83$ & 36.0 \\
\hline
\end{tabular}


Table 3. Observing opportunities for NEAs with highest predicted Yarkovsky drift rates. Apparitions listed are when elongation is greater than $90^{\circ}$. Apparitions with magnitude ranges always above $23.5 \mathrm{~V}(\mathrm{mag})$ are not shown.

\begin{tabular}{|c|c|c|c|c|c|}
\hline NEA & $\begin{array}{c}d a / d t \\
10^{-4} \mathrm{AU} / \mathrm{Myr}\end{array}$ & \multicolumn{2}{|l|}{ Apparition } & $V$ (mag) range & $\begin{array}{r}\text { Dec range } \\
\text { degrees }\end{array}$ \\
\hline 2010 JG87 & $64.99 \pm 23.26$ & May 292014 & - $\quad$ Sep 202014 & $20.3,24.4$ & 07,19 \\
\hline 2006 HY51 & $34.32 \pm 14.86$ & Feb 082014 & - Jul 062014 & $21.5,23.6$ & $-12,-03$ \\
\hline & & Feb 042015 & - $\quad$ Jun 172015 & $20.4,22.9$ & 06,22 \\
\hline & & Feb 052018 & - $\quad$ Jul 082018 & $22.3,24.0$ & $-10,-02$ \\
\hline & & Mar 112019 & - $\quad$ Jun 052019 & $17.9,21.8$ & 24,53 \\
\hline & & Jan 252020 & - Jul 012020 & $23.4,24.6$ & 00,08 \\
\hline & & Feb 032022 & - $\quad$ Apr 012022 & $23.3,24.3$ & $-09,-06$ \\
\hline 2007 EP88 & $31.04 \pm 12.04$ & Mar 142013 & - $\quad$ May 142013 & $17.2,21.2$ & $-58,-03$ \\
\hline & & Feb 092014 & - $\quad$ May 262014 & $19.7,21.1$ & $-44,-14$ \\
\hline & & Mar 112015 & - $\quad$ Mar 252015 & $19.9,20.6$ & $-59,-51$ \\
\hline & & Feb 102017 & - $\quad$ May 262017 & $19.5,21.2$ & $-42,-11$ \\
\hline & & Feb 192018 & - $\quad$ Apr 272018 & $18.8,21.3$ & $-79,-40$ \\
\hline & & Feb 172020 & - $\quad$ May 242020 & $19.0,21.4$ & $-43,-09$ \\
\hline & & Feb 132021 & - $\quad$ May 142021 & $19.2,21.4$ & $-56,-27$ \\
\hline \multirow[t]{8}{*}{ (137924) 2000 BD19 } & $34.30 \pm 12.02$ & Dec 152012 & - $\quad$ Mar 102013 & $17.8,20.2$ & 42,76 \\
\hline & & Nov 282013 & - $\quad$ Mar 172014 & $18.5,19.9$ & 31,53 \\
\hline & & Dec 052014 & - $\quad$ Feb 282015 & $16.6,20.2$ & 23,54 \\
\hline & & Jan 232017 & - $\quad$ Feb 192017 & $18.2,19.4$ & 46,54 \\
\hline & & Dec 032017 & - $\quad$ Mar 152018 & $18.4,20.1$ & 36,61 \\
\hline & & Nov 292018 & - $\quad$ Mar 152019 & $18.1,20.2$ & 27,50 \\
\hline & & Dec 142019 & - $\quad$ Feb 052020 & $15.7,19.8$ & 16,22 \\
\hline & & Dec 222021 & - $\quad$ Mar 082022 & $17.6,20.1$ & 43,85 \\
\hline \multirow{8}{*}{ (153201) 2000 WO107 } & $69.83 \pm 30.39$ & Apr 282015 & - $\quad$ May 192015 & $22.7,23.3$ & 14,26 \\
\hline & $57.95 \pm 20.60$ & May 222013 & - $\quad$ Jul 162013 & $16.6,21.0$ & $-10,-04$ \\
\hline & & Jun 102016 & - $\quad$ Jul 132016 & $20.6,22.1$ & $-06,08$ \\
\hline & $23.45 \pm 10.14$ & Dec 152013 & - $\quad$ Feb 062014 & $18.7,21.8$ & $-02,20$ \\
\hline & & Nov 082014 & - $\quad$ Feb 262015 & $18.9,22.2$ & 17,31 \\
\hline & & Nov 052015 & - $\quad$ Mar 042016 & $19.7,21.8$ & 20,34 \\
\hline & & Nov 132016 & - $\quad$ Feb 252017 & $18.9,22.2$ & 19,44 \\
\hline & & Dec 092017 & - $\quad$ Jan 102018 & $19.9,21.5$ & 08,13 \\
\hline
\end{tabular}


Table 3-Continued

\begin{tabular}{|c|c|c|c|c|c|c|}
\hline NEA & $\begin{array}{c}d a / d t \\
10^{-4} \mathrm{AU} / \mathrm{Myr}\end{array}$ & Apparition & & & $V$ (mag) range & $\begin{array}{r}\text { Dec range } \\
\text { degrees }\end{array}$ \\
\hline & & Nov 292020 & - & Feb 142021 & $13.0,22.1$ & 13,25 \\
\hline & & Nov 052021 & - & Mar 012022 & $19.3,22.1$ & 19,32 \\
\hline 2010 EX11 & $89.47 \pm 40.33$ & (not observable) & & & & \\
\hline \multirow[t]{4}{*}{2008 EY5 } & $31.79 \pm 10.60$ & Feb 252013 & - & Mar 232013 & $17.4,19.1$ & $-76,-46$ \\
\hline & & Feb 252014 & - & Mar 202014 & $17.6,19.4$ & $-85,-45$ \\
\hline & & Feb 272015 & - & Mar 172015 & $18.2,19.5$ & $-69,-43$ \\
\hline & & Mar 022016 & - & Mar 102016 & $19.1,19.6$ & $-51,-43$ \\
\hline \multirow[t]{5}{*}{$2006 \mathrm{NL}$} & $20.05 \pm 12.66$ & Jun 302013 & - & Oct 022013 & $16.7,21.8$ & $-42,19$ \\
\hline & & Jul 152014 & - & Sep 052014 & $20.3,21.8$ & $-30,-08$ \\
\hline & & Jun 212017 & - & Oct 092017 & $19.5,21.2$ & $-60,-04$ \\
\hline & & Jul 072020 & - & Sep 272020 & $15.4,21.8$ & $-38,62$ \\
\hline & & Jul 062021 & - & Sep 182021 & $19.9,21.8$ & $-54,-08$ \\
\hline \multirow[t]{4}{*}{2006 MD12 } & $19.60 \pm 12.15$ & May 222012 & - & Aug 302012 & $18.6,21.3$ & $-61,-15$ \\
\hline & & Jun 122015 & - & Aug 201015 & $19.4,21.5$ & $-61,-24$ \\
\hline & & May 252016 & - & Aug 152016 & $18.3,21.5$ & $-15,55$ \\
\hline & & May 182019 & - & Aug 312019 & $17.8,21.2$ & $-28,02$ \\
\hline \multirow[t]{2}{*}{2010 GQ75 } & $36.30 \pm 12.49$ & Apr 142013 & - & Sep 022013 & $23.3,25.7$ & $-53,-32$ \\
\hline & & Apr 302017 & - & Jul 252017 & $21.5,24.5$ & $-70,-36$ \\
\hline
\end{tabular}


Table 4. NEAs with highest predicted Yarkovsky drift rates

\begin{tabular}{|c|c|c|c|c|c|c|c|c|}
\hline NEA & $\begin{array}{c}a \\
(\mathrm{AU})\end{array}$ & $e$ & $\begin{array}{c}i \\
(\mathrm{deg})\end{array}$ & $\begin{array}{l}D \\
\qquad(\mathrm{~km})\end{array}$ & $p_{V}$ & Arc & $\begin{array}{c}d a / d t \\
10^{-4} \mathrm{AU} / \mathrm{Myr}\end{array}$ & $\begin{array}{l}\Delta \rho \\
(\mathrm{km})\end{array}$ \\
\hline 2010 JG87 & 2.76 & 0.95 & 16.91 & $0.41 \pm 0.02$ & $0.20 \pm 0.04$ & 54 days & $72.11 \pm 25.12$ & 110.0 \\
\hline 2006 HY51 & 2.60 & 0.97 & 30.58 & $1.22 \pm 0.27$ & $0.16 \pm 0.09$ & $2006-2011$ & $54.35 \pm 23.85$ & 90.8 \\
\hline 2007 EP88 & 0.84 & 0.89 & 20.78 & $0.64 \pm 0.04$ & $0.17 \pm 0.04$ & $2007-2010$ & $48.57 \pm 16.22$ & 443.8 \\
\hline (137924) 2000 BD19 & 0.88 & 0.89 & 25.69 & $0.97 \pm 0.04$ & $0.25 \pm 0.05$ & $1997-2010$ & $34.30 \pm 12.02$ & 292.6 \\
\hline 2010 HX107 & 0.80 & 0.30 & 3.37 & $0.06 \pm 0.01$ & $0.19 \pm 0.07$ & 60 days & $33.11 \pm 23.05$ & 323.6 \\
\hline 2002 LT24 & 0.72 & 0.50 & 0.76 & $0.14 \pm 0.02$ & $0.14 \pm 0.07$ & $2002-2010$ & $29.06 \pm 16.58$ & 333.1 \\
\hline (153201) 2000 WO107 & 0.91 & 0.78 & 7.78 & $0.51 \pm 0.08$ & $0.13 \pm 0.06$ & $2000-2010$ & $23.45 \pm 10.14$ & 188.7 \\
\hline 2010 EX11 & 0.96 & 0.11 & 9.75 & $0.04 \pm 0.01$ & $0.23 \pm 0.09$ & 37 days & $23.09 \pm 18.64$ & 173.0 \\
\hline 2008 EY5 & 0.63 & 0.63 & 5.07 & $0.36 \pm 0.01$ & $0.12 \pm 0.03$ & 2008-2011 & $23.00 \pm 9.75$ & 324.6 \\
\hline $2006 \mathrm{NL}$ & 0.85 & 0.58 & 20.08 & $0.22 \pm 0.05$ & $0.46 \pm 0.14$ & 2006-2009 & $20.05 \pm 12.66$ & 179.8 \\
\hline 2006 MD12 & 0.84 & 0.61 & 27.27 & $0.27 \pm 0.06$ & $0.43 \pm 0.13$ & 2006-2009 & $19.60 \pm 12.15$ & 178.6 \\
\hline 2010 GQ75 & 2.43 & 0.87 & 43.23 & $0.37 \pm 0.02$ & $0.11 \pm 0.03$ & 34 days & $19.48 \pm 9.83$ & 36.0 \\
\hline 2010 GR7 & 1.83 & 0.85 & 24.22 & $0.45 \pm 0.01$ & $0.38 \pm 0.07$ & $2010-2011$ & $19.33 \pm 9.42$ & 54.6 \\
\hline 2001 CQ36 & 0.94 & 0.18 & 1.29 & $0.07 \pm 0.01$ & $0.41 \pm 0.11$ & 2001-2011 & $19.13 \pm 13.63$ & 146.9 \\
\hline 2010 AJ30 & 0.81 & 0.30 & 7.59 & $0.11 \pm 0.02$ & $0.12 \pm 0.06$ & 37 days & $18.61 \pm 12.30$ & 178.1 \\
\hline 2004 LG & 2.07 & 0.90 & 70.97 & $0.87 \pm 0.01$ & $0.15 \pm 0.03$ & 2004-2010 & $17.26 \pm 7.47$ & 40.7 \\
\hline 2010 HW81 & 1.22 & 0.73 & 12.85 & $0.35 \pm 0.05$ & $0.04 \pm 0.02$ & 10 days & $16.08 \pm 8.64$ & 83.8 \\
\hline 2010 NB2 & 2.08 & 0.76 & 28.66 & $0.21 \pm 0.01$ & $0.30 \pm 0.05$ & 34 days & $14.09 \pm 9.16$ & 32.9 \\
\hline 2010 NJ1 & 0.97 & 0.54 & 11.22 & $0.22 \pm 0.04$ & $0.26 \pm 0.10$ & $2010-2011$ & $13.97 \pm 8.77$ & 102.6 \\
\hline (252399) 2001 TX44 & 0.87 & 0.55 & 15.21 & $0.26 \pm 0.05$ & $0.68 \pm 0.20$ & $2001-2010$ & $13.81 \pm 9.00$ & 118.2 \\
\hline $2010 \mathrm{GA} 7$ & 0.91 & 0.39 & 30.02 & $0.15 \pm 0.02$ & $0.26 \pm 0.12$ & 54 days & $13.57 \pm 9.25$ & 108.9 \\
\hline (162195) 1999 RK45 & 1.60 & 0.77 & 5.89 & $0.39 \pm 0.08$ & $0.19 \pm 0.06$ & $1999-2011$ & $13.39 \pm 8.38$ & 46.4 \\
\hline (139289) $2001 \mathrm{KR} 1$ & 1.26 & 0.84 & 23.23 & $1.13 \pm 0.24$ & $0.13 \pm 0.07$ & $2001-2010$ & $12.98 \pm 7.36$ & 64.3 \\
\hline 2005 GB120 & 0.79 & 0.39 & 9.15 & $0.21 \pm 0.01$ & $0.28 \pm 0.04$ & $2005-2010$ & $12.44 \pm 7.53$ & 123.7 \\
\hline 2006 SY5 & 1.04 & 0.15 & 7.56 & $0.09 \pm 0.02$ & $0.34 \pm 0.23$ & $2006-2007$ & $12.19 \pm 9.89$ & 80.1 \\
\hline 2010 NU1 & 2.12 & 0.78 & 33.70 & $0.29 \pm 0.05$ & $0.07 \pm 0.03$ & 8 days & $11.76 \pm 7.57$ & 26.7 \\
\hline $2005 \mathrm{MB}$ & 0.99 & 0.79 & 41.40 & $1.01 \pm 0.14$ & $0.25 \pm 0.10$ & $2003-2011$ & $11.66 \pm 5.05$ & 83.5 \\
\hline (234341) 2001 FZ57 & 0.94 & 0.60 & 20.67 & $0.34 \pm 0.05$ & $0.49 \pm 0.19$ & $2000-2011$ & $11.58 \pm 6.71$ & 88.4 \\
\hline 2010 LJ61 & 1.04 & 0.46 & 9.73 & $0.19 \pm 0.03$ & $0.21 \pm 0.08$ & 10 days & $11.02 \pm 8.22$ & 72.6 \\
\hline (225312) 1996 XB27 & 1.19 & 0.06 & 2.47 & $0.08 \pm 0.02$ & $0.48 \pm 0.26$ & 1996-2009 & $10.63 \pm 8.81$ & 57.4 \\
\hline
\end{tabular}


Table 4-Continued

\begin{tabular}{|c|c|c|c|c|c|c|c|c|}
\hline NEA & $\begin{array}{c}a \\
(\mathrm{AU})\end{array}$ & $e$ & $\begin{array}{c}i \\
(\operatorname{deg})\end{array}$ & $\begin{array}{l}D \\
\quad(\mathrm{~km})\end{array}$ & $p_{V}$ & Arc & $\begin{array}{c}d a / d t \\
10^{-4} \mathrm{AU} / \mathrm{Myr}\end{array}$ & $\begin{array}{l}\Delta \rho \\
(\mathrm{km})\end{array}$ \\
\hline 2008 EM9 & 1.96 & 0.85 & 9.40 & $0.79 \pm 0.03$ & $0.32 \pm 0.07$ & $2008-2011$ & $10.60 \pm 5.22$ & 27.0 \\
\hline (66400) 1999 LT7 & 0.86 & 0.57 & 9.07 & $0.41 \pm 0.08$ & $0.18 \pm 0.09$ & $1999-2010$ & $10.25 \pm 5.93$ & 90.7 \\
\hline $2008 \mathrm{CN} 1$ & 0.77 & 0.35 & 7.22 & $0.23 \pm 0.00$ & $0.20 \pm 0.03$ & $2008-2010$ & $9.93 \pm 6.71$ & 102.8 \\
\hline (164202) $2004 \mathrm{EW}$ & 0.99 & 0.28 & 4.66 & $0.16 \pm 0.03$ & $0.36 \pm 0.22$ & $2004-2011$ & $8.97 \pm 6.47$ & 63.9 \\
\hline 2010 NG1 & 0.85 & 0.33 & 24.74 & $0.23 \pm 0.04$ & $0.23 \pm 0.11$ & 71 days & $8.96 \pm 6.03$ & 80.1 \\
\hline (141531) 2002 GB & 0.99 & 0.53 & 22.56 & $0.30 \pm 0.01$ & $0.48 \pm 0.08$ & $2002-2012$ & $8.91 \pm 5.37$ & 63.1 \\
\hline $2009 \mathrm{JO} 2$ & 0.89 & 0.48 & 19.63 & $0.31 \pm 0.03$ & $0.34 \pm 0.09$ & 2009-2011 & $8.85 \pm 5.55$ & 74.0 \\
\hline (255071) 2005 UH6 & 1.00 & 0.63 & 2.64 & $0.52 \pm 0.14$ & $0.22 \pm 0.07$ & $2005-2011$ & $8.83 \pm 6.11$ & 61.7 \\
\hline 2010 FA81 & 1.20 & 0.15 & 15.48 & $0.10 \pm 0.02$ & $0.22 \pm 0.09$ & 48 days & $8.75 \pm 7.80$ & 46.9 \\
\hline (33342) 1998 WT24 & 0.72 & 0.42 & 7.34 & $0.42 \pm 0.04$ & $0.56 \pm 0.20$ & $1998-2008$ & $8.70 \pm 4.93$ & 100.0 \\
\hline 1998 SV4 & 0.82 & 0.64 & 53.30 & $0.74 \pm 0.15$ & $0.20 \pm 0.06$ & $1998-2010$ & $8.53 \pm 4.40$ & 80.9 \\
\hline (163758) 2003 OS13 & 1.30 & 0.74 & 41.56 & $0.66 \pm 0.13$ & $0.44 \pm 0.14$ & 2003-2009 & $8.52 \pm 5.15$ & 40.4 \\
\hline 2010 LH14 & 2.20 & 0.57 & 4.66 & $0.10 \pm 0.02$ & $0.27 \pm 0.11$ & 152 days & $8.30 \pm 7.22$ & 17.8 \\
\hline (307918) 2004 EU9 & 0.88 & 0.51 & 28.59 & $0.37 \pm 0.05$ & $0.36 \pm 0.10$ & $2004-2010$ & $8.22 \pm 5.21$ & 69.7 \\
\hline (267221) 2001 AD2 & 1.04 & 0.66 & 1.66 & $0.56 \pm 0.02$ & $0.10 \pm 0.02$ & $2001-2011$ & $8.08 \pm 4.37$ & 53.4 \\
\hline $2006 \mathrm{KZ112}$ & 2.52 & 0.89 & 37.76 & $1.18 \pm 0.03$ & $0.27 \pm 0.05$ & $2006-2010$ & $8.06 \pm 3.89$ & 14.1 \\
\hline 2003 HB & 0.85 & 0.38 & 18.11 & $0.29 \pm 0.05$ & $0.27 \pm 0.10$ & $2003-2010$ & $7.79 \pm 5.01$ & 69.6 \\
\hline 2010 GW62 & 1.27 & 0.58 & 32.43 & $0.28 \pm 0.02$ & $0.38 \pm 0.08$ & 72 days & $7.62 \pm 5.19$ & 37.3 \\
\hline $2010 \mathrm{JE} 87$ & 0.91 & 0.44 & 17.15 & $0.31 \pm 0.02$ & $0.11 \pm 0.03$ & 5 days & $7.42 \pm 4.82$ & 60.1 \\
\hline (164207) 2004 GU9 & 1.00 & 0.14 & 13.65 & $0.16 \pm 0.01$ & $0.22 \pm 0.04$ & $2001-2011$ & $7.26 \pm 6.21$ & 50.8 \\
\hline (275974) $2001 \mathrm{XD}$ & 2.04 & 0.80 & 11.41 & $0.63 \pm 0.10$ & $0.22 \pm 0.10$ & $2001-2010$ & $7.06 \pm 4.41$ & 16.9 \\
\hline 2010 NY65 & 1.00 & 0.37 & 11.72 & $0.23 \pm 0.01$ & $0.07 \pm 0.01$ & 27 days & $6.93 \pm 4.75$ & 48.9 \\
\hline 2010 LK68 & 1.16 & 0.47 & 21.76 & $0.24 \pm 0.02$ & $0.03 \pm 0.01$ & 4 days & $6.86 \pm 4.57$ & 38.3 \\
\hline (141432) 2002 CQ11 & 0.98 & 0.43 & 2.46 & $0.24 \pm 0.04$ & $0.34 \pm 0.14$ & $2002-2011$ & $6.83 \pm 5.12$ & 49.4 \\
\hline 2009 WZ104 & 0.86 & 0.19 & 9.84 & $0.24 \pm 0.00$ & $0.31 \pm 0.07$ & $2005-2010$ & $6.59 \pm 4.72$ & 58.3 \\
\hline (162269) 1999 VO6 & 1.14 & 0.74 & 40.10 & $1.01 \pm 0.18$ & $0.27 \pm 0.11$ & $1993-2011$ & $6.56 \pm 3.69$ & 37.9 \\
\hline 2010 DK34 & 2.75 & 0.76 & 27.47 & $0.28 \pm 0.01$ & $0.15 \pm 0.03$ & 32 days & $6.53 \pm 4.84$ & 10.0 \\
\hline (41429) 2000 GE2 & 1.59 & 0.55 & 2.19 & $0.20 \pm 0.03$ & $0.27 \pm 0.08$ & $1998-2010$ & $6.53 \pm 5.07$ & 22.7 \\
\hline (225416) $1999 \mathrm{YC}$ & 1.42 & 0.83 & 38.22 & $1.65 \pm 0.18$ & $0.09 \pm 0.03$ & $1999-2010$ & $6.48 \pm 2.77$ & 26.8 \\
\hline (67381) 2000 OL8 & 1.32 & 0.54 & 10.67 & $0.28 \pm 0.06$ & $0.25 \pm 0.08$ & $2000-2011$ & $6.43 \pm 4.96$ & 29.7 \\
\hline
\end{tabular}


Table 4-Continued

\begin{tabular}{|c|c|c|c|c|c|c|c|c|}
\hline NEA & $\begin{array}{c}a \\
(\mathrm{AU})\end{array}$ & $e$ & $\begin{array}{c}i \\
(\operatorname{deg})\end{array}$ & $\begin{array}{l}D \\
\qquad(\mathrm{~km})\end{array}$ & $p_{V}$ & Arc & $\begin{array}{c}d a / d t \\
10^{-4} \mathrm{AU} / \mathrm{Myr}\end{array}$ & $\begin{array}{l}\Delta \rho \\
(\mathrm{km})\end{array}$ \\
\hline 2010 KU7 & 1.66 & 0.38 & 6.76 & $0.10 \pm 0.01$ & $0.22 \pm 0.08$ & 40 days & $6.42 \pm 5.06$ & 20.9 \\
\hline (152564) $1992 \mathrm{HF}$ & 1.39 & 0.56 & 13.31 & $0.28 \pm 0.05$ & $0.17 \pm 0.07$ & $1992-2010$ & $6.40 \pm 4.77$ & 27.3 \\
\hline 2006 DS14 & 0.86 & 0.34 & 26.53 & $0.32 \pm 0.01$ & $0.12 \pm 0.02$ & $2002-2010$ & $6.26 \pm 4.00$ & 54.6 \\
\hline 2010 GR75 & 1.73 & 0.64 & 17.78 & $0.28 \pm 0.05$ & $0.33 \pm 0.09$ & 84 days & $6.23 \pm 4.47$ & 19.2 \\
\hline (242191) 2003 NZ6 & 0.79 & 0.49 & 18.24 & $0.37 \pm 0.03$ & $0.33 \pm 0.07$ & $2003-2010$ & $6.13 \pm 4.07$ & 60.7 \\
\hline $2002 \mathrm{WZ2}$ & 2.46 & 0.88 & 51.40 & $1.60 \pm 0.12$ & $0.11 \pm 0.03$ & $2002-2010$ & $6.10 \pm 3.12$ & 11.1 \\
\hline (230111) 2001 BE10 & 0.82 & 0.37 & 17.51 & $0.40 \pm 0.06$ & $0.25 \pm 0.06$ & 2001-2010 & $6.09 \pm 4.26$ & 57.0 \\
\hline $2005 \mathrm{WC} 1$ & 1.40 & 0.49 & 19.98 & $0.29 \pm 0.06$ & $0.11 \pm 0.03$ & $2005-2011$ & $6.00 \pm 4.25$ & 25.3 \\
\hline (242643) 2005 NZ6 & 1.83 & 0.86 & 8.50 & $1.99 \pm 0.48$ & $0.04 \pm 0.04$ & $2005-2010$ & $5.95 \pm 3.48$ & 16.8 \\
\hline $2010 \mathrm{JG}$ & 1.19 & 0.32 & 23.31 & $0.19 \pm 0.03$ & $0.21 \pm 0.08$ & $2010-2011$ & $5.88 \pm 4.90$ & 31.7 \\
\hline (184990) $2006 \mathrm{KE} 89$ & 1.05 & 0.80 & 45.08 & $1.96 \pm 0.11$ & $0.13 \pm 0.03$ & 1994-2012 & $5.79 \pm 2.53$ & 37.5 \\
\hline 2010 FG81 & 1.66 & 0.39 & 7.97 & $0.11 \pm 0.00$ & $0.07 \pm 0.01$ & 36 days & $5.78 \pm 4.45$ & 18.9 \\
\hline $2010 \mathrm{KX} 7$ & 0.99 & 0.17 & 21.48 & $0.21 \pm 0.02$ & $0.08 \pm 0.02$ & $2010-2011$ & $5.71 \pm 4.57$ & 40.6 \\
\hline 2009 WA & 1.14 & 0.14 & 29.84 & $0.16 \pm 0.03$ & $0.22 \pm 0.14$ & 174 days & $5.68 \pm 4.51$ & 32.8 \\
\hline 2010 OC101 & 1.22 & 0.23 & 13.60 & $0.15 \pm 0.00$ & $0.38 \pm 0.07$ & 182 days & $5.66 \pm 3.95$ & 29.4 \\
\hline (40267) 1999 GJ4 & 1.34 & 0.81 & 34.53 & $1.64 \pm 0.05$ & $0.45 \pm 0.09$ & $1955-2009$ & $5.49 \pm 2.49$ & 24.8 \\
\hline 2010 HZ104 & 2.25 & 0.57 & 20.22 & $0.14 \pm 0.02$ & $0.09 \pm 0.06$ & 23 days & $5.43 \pm 4.51$ & 11.2 \\
\hline 2010 LU108 & 2.24 & 0.82 & 9.51 & $0.82 \pm 0.01$ & $0.03 \pm 0.01$ & 51 days & $5.37 \pm 3.11$ & 11.2 \\
\hline 2010 OF101 & 0.95 & 0.33 & 23.37 & $0.30 \pm 0.05$ & $0.23 \pm 0.12$ & $2010-2011$ & $5.26 \pm 3.69$ & 39.7 \\
\hline $2010 \mathrm{HC}$ & 2.13 & 0.52 & 6.88 & $0.13 \pm 0.01$ & $0.33 \pm 0.06$ & 114 days & $5.25 \pm 4.50$ & 11.8 \\
\hline (285339) 1999 JR6 & 1.37 & 0.68 & 20.34 & $0.61 \pm 0.02$ & $0.27 \pm 0.06$ & $1999-2011$ & $5.25 \pm 3.27$ & 23.0 \\
\hline 2003 CR1 & 1.45 & 0.46 & 12.71 & $0.20 \pm 0.03$ & $0.43 \pm 0.15$ & $2003-2010$ & $5.20 \pm 3.53$ & 20.8 \\
\hline (192559) $1998 \mathrm{VO}$ & 1.07 & 0.23 & 10.06 & $0.22 \pm 0.04$ & $0.30 \pm 0.17$ & $1998-2008$ & $5.15 \pm 4.43$ & 32.4 \\
\hline (194268) 2001 UY4 & 1.45 & 0.79 & 5.43 & $1.24 \pm 0.02$ & $0.05 \pm 0.01$ & $2001-2011$ & $5.13 \pm 2.54$ & 20.5 \\
\hline 2010 KY127 & 2.50 & 0.88 & 60.38 & $1.77 \pm 0.29$ & $0.13 \pm 0.06$ & $2010-2011$ & $5.12 \pm 2.56$ & 9.1 \\
\hline 2010 CJ171 & 2.00 & 0.49 & 7.38 & $0.13 \pm 0.00$ & $0.36 \pm 0.07$ & 174 days & $5.12 \pm 4.50$ & 12.7 \\
\hline (277570) 2005 YP180 & 1.37 & 0.62 & 4.11 & $0.44 \pm 0.05$ & $0.18 \pm 0.04$ & $2005-2011$ & $5.09 \pm 3.37$ & 22.2 \\
\hline 2002 GO5 & 1.90 & 0.77 & 13.81 & $0.75 \pm 0.15$ & $0.24 \pm 0.08$ & $2002-2004$ & $5.08 \pm 3.37$ & 13.6 \\
\hline 2005 YY93 & 2.58 & 0.88 & 23.43 & $1.75 \pm 0.06$ & $0.08 \pm 0.02$ & $2005-2010$ & $5.03 \pm 2.47$ & 8.5 \\
\hline 2010 OS22 & 1.64 & 0.69 & 9.36 & $0.50 \pm 0.01$ & $0.41 \pm 0.09$ & $2010-2011$ & $5.01 \pm 3.02$ & 16.8 \\
\hline
\end{tabular}


Table 4-Continued

\begin{tabular}{|c|c|c|c|c|c|c|c|c|}
\hline NEA & $\begin{array}{c}a \\
(\mathrm{AU})\end{array}$ & $e$ & $\begin{array}{c}i \\
(\operatorname{deg})\end{array}$ & $\begin{array}{l}D \\
\qquad(\mathrm{~km})\end{array}$ & $p_{V}$ & Arc & $\begin{array}{c}d a / d t \\
10^{-4} \mathrm{AU} / \mathrm{Myr}\end{array}$ & $\begin{array}{c}\Delta \rho \\
(\mathrm{km})\end{array}$ \\
\hline (250680) 2005 QC5 & 0.89 & 0.36 & 9.45 & $0.40 \pm 0.01$ & $0.15 \pm 0.03$ & $1978-2011$ & $4.94 \pm 3.33$ & 41.0 \\
\hline 2010 LL68 & 2.07 & 0.53 & 10.49 & $0.15 \pm 0.02$ & $0.06 \pm 0.02$ & 124 days & $4.93 \pm 4.16$ & 11.6 \\
\hline 2010 PM58 & 1.37 & 0.46 & 13.60 & $0.26 \pm 0.04$ & $0.10 \pm 0.04$ & 181 days & $4.80 \pm 3.76$ & 20.9 \\
\hline $2007 \mathrm{VD} 12$ & 1.15 & 0.36 & 22.86 & $0.22 \pm 0.04$ & $0.39 \pm 0.12$ & 19 days & $4.63 \pm 3.40$ & 26.4 \\
\hline 1999 GY5 & 1.15 & 0.61 & 24.44 & $0.67 \pm 0.12$ & $0.04 \pm 0.02$ & $1999-2010$ & $4.59 \pm 2.90$ & 26.2 \\
\hline 2010 PU66 & 1.49 & 0.39 & 18.09 & $0.18 \pm 0.01$ & $0.08 \pm 0.02$ & 62 days & $4.58 \pm 3.87$ & 17.7 \\
\hline (312070) 2007 TA19 & 0.95 & 0.51 & 22.63 & $0.60 \pm 0.10$ & $0.09 \pm 0.04$ & $2007-2011$ & $4.55 \pm 2.82$ & 34.2 \\
\hline 2010 CB55 & 1.13 & 0.15 & 25.92 & $0.20 \pm 0.00$ & $0.04 \pm 0.01$ & 189 days & $4.51 \pm 3.42$ & 26.2 \\
\hline 2010 JK33 & 2.23 & 0.61 & 4.03 & $0.21 \pm 0.01$ & $0.15 \pm 0.03$ & 2010-2010 & $4.50 \pm 3.63$ & 9.5 \\
\hline 2010 GO33 & 2.41 & 0.70 & 19.12 & $0.31 \pm 0.02$ & $0.38 \pm 0.09$ & $2010-2010$ & $4.49 \pm 3.55$ & 8.4 \\
\hline (152754) 1999 GS6 & 1.19 & 0.50 & 2.02 & $0.41 \pm 0.08$ & $0.22 \pm 0.11$ & $1999-2010$ & $4.43 \pm 3.37$ & 23.9 \\
\hline (4034) Vishnu & 1.06 & 0.44 & 11.17 & $0.42 \pm 0.07$ & $0.58 \pm 0.25$ & 1986-2009 & $4.37 \pm 3.00$ & 28.1 \\
\hline 2010 GT7 & 2.71 & 0.68 & 9.28 & $0.22 \pm 0.01$ & $0.33 \pm 0.06$ & 75 days & $4.37 \pm 2.95$ & 6.8 \\
\hline $2010 \mathrm{FL}$ & 1.91 & 0.66 & 11.45 & $0.35 \pm 0.01$ & $0.27 \pm 0.05$ & $2002-2010$ & $4.37 \pm 3.20$ & 11.5 \\
\hline 2002 LS32 & 1.78 & 0.70 & 8.83 & $0.57 \pm 0.12$ & $0.29 \pm 0.09$ & $2002-2010$ & $4.35 \pm 3.58$ & 12.8 \\
\hline (152671) 1998 HL3 & 1.13 & 0.37 & 2.68 & $0.30 \pm 0.01$ & $0.20 \pm 0.04$ & $1998-2012$ & $4.31 \pm 3.22$ & 25.2 \\
\hline 2007 BM8 & 1.34 & 0.72 & 27.63 & $1.08 \pm 0.17$ & $0.07 \pm 0.02$ & $2002-2010$ & $4.26 \pm 2.52$ & 19.2 \\
\hline 2010 FH81 & 1.23 & 0.21 & 16.79 & $0.20 \pm 0.01$ & $0.09 \pm 0.02$ & 107 days & $4.21 \pm 3.43$ & 21.7 \\
\hline 2010 OE22 & 2.65 & 0.63 & 14.31 & $0.17 \pm 0.01$ & $0.18 \pm 0.04$ & 61 days & $4.20 \pm 3.17$ & 6.8 \\
\hline 2010 CC55 & 1.55 & 0.47 & 6.79 & $0.24 \pm 0.01$ & $0.03 \pm 0.01$ & 2010-2011 & $4.15 \pm 3.08$ & 15.1 \\
\hline 2010 GS7 & 2.71 & 0.65 & 9.68 & $0.20 \pm 0.01$ & $0.11 \pm 0.03$ & 154 days & $4.04 \pm 3.15$ & 6.3 \\
\hline 2010 LM14 & 1.11 & 0.38 & 25.92 & $0.36 \pm 0.08$ & $0.04 \pm 0.01$ & $2010-2011$ & $3.98 \pm 3.18$ & 23.8 \\
\hline 2010 GF 25 & 1.40 & 0.73 & 37.57 & $1.13 \pm 0.10$ & $0.02 \pm 0.01$ & 8 days & $3.93 \pm 2.22$ & 16.7 \\
\hline 2010 OB101 & 1.62 & 0.52 & 9.12 & $0.28 \pm 0.02$ & $0.14 \pm 0.03$ & 2010-2011 & $3.90 \pm 2.98$ & 13.3 \\
\hline 2010 LE15 & 0.86 & 0.27 & 13.26 & $0.46 \pm 0.01$ & $0.15 \pm 0.02$ & $2001-2010$ & $3.86 \pm 2.72$ & 33.6 \\
\hline 2010 LJ68 & 1.69 & 0.43 & 16.50 & $0.19 \pm 0.04$ & $0.04 \pm 0.02$ & 7 days & $3.85 \pm 2.86$ & 12.3 \\
\hline (152637) $1997 \mathrm{NC} 1$ & 0.87 & 0.21 & 16.72 & $0.43 \pm 0.10$ & $0.59 \pm 0.20$ & 1997-2009 & $3.83 \pm 2.90$ & 33.3 \\
\hline 2010 OL100 & 2.26 & 0.65 & 22.16 & $0.31 \pm 0.04$ & $0.25 \pm 0.06$ & 160 days & $3.74 \pm 2.86$ & 7.7 \\
\hline 2002 EZ2 & 1.25 & 0.05 & 13.02 & $0.21 \pm 0.04$ & $0.40 \pm 0.12$ & $2002-2009$ & $3.67 \pm 3.72$ & 18.4 \\
\hline 2010 GK23 & 2.92 & 0.70 & 35.38 & $0.28 \pm 0.04$ & $0.30 \pm 0.13$ & 8 days & $3.62 \pm 3.01$ & 5.1 \\
\hline
\end{tabular}


Table 4-Continued

\begin{tabular}{|c|c|c|c|c|c|c|c|c|}
\hline NEA & $\begin{array}{c}a \\
(\mathrm{AU})\end{array}$ & $e$ & $\begin{array}{c}i \\
(\operatorname{deg})\end{array}$ & $\begin{array}{l}D \\
\qquad(\mathrm{~km})\end{array}$ & $p_{V}$ & Arc & $\begin{array}{c}d a / d t \\
10^{-4} \mathrm{AU} / \mathrm{Myr}\end{array}$ & $\begin{array}{c}\Delta \rho \\
(\mathrm{km})\end{array}$ \\
\hline 2005 OU2 & 1.23 & 0.37 & 47.77 & $0.34 \pm 0.07$ & $0.37 \pm 0.11$ & 2005-2009 & $3.57 \pm 2.92$ & 18.2 \\
\hline (6239) Minos & 1.15 & 0.41 & 3.95 & $0.47 \pm 0.12$ & $0.56 \pm 0.39$ & 1983-2009 & $3.54 \pm 2.68$ & 20.0 \\
\hline 2010 GP67 & 1.11 & 0.11 & 13.27 & $0.25 \pm 0.02$ & $0.03 \pm 0.01$ & 205 days & $3.53 \pm 2.71$ & 21.0 \\
\hline $2009 \mathrm{XF} 2$ & 1.29 & 0.25 & 14.01 & $0.24 \pm 0.04$ & $0.27 \pm 0.14$ & 202 days & $3.52 \pm 3.19$ & 16.9 \\
\hline 2010 JD87 & 1.43 & 0.64 & 24.58 & $0.69 \pm 0.01$ & $0.09 \pm 0.02$ & 28 days & $3.50 \pm 2.23$ & 14.4 \\
\hline 2010 OH126 & 1.90 & 0.50 & 14.39 & $0.22 \pm 0.01$ & $0.10 \pm 0.02$ & 15 days & $3.49 \pm 2.75$ & 9.3 \\
\hline (152941) 2000 FM10 & 1.48 & 0.68 & 8.74 & $0.82 \pm 0.00$ & $0.21 \pm 0.03$ & $2000-2012$ & $3.49 \pm 2.23$ & 13.5 \\
\hline (3361) Orpheus & 1.21 & 0.32 & 2.69 & $0.35 \pm 0.06$ & $0.36 \pm 0.13$ & $1982-2009$ & $3.44 \pm 2.41$ & 18.1 \\
\hline 2010 LV108 & 2.75 & 0.66 & 4.78 & $0.23 \pm 0.01$ & $0.03 \pm 0.00$ & 30 days & $3.44 \pm 2.64$ & 5.3 \\
\hline (163243) 2002 FB3 & 0.76 & 0.60 & 20.27 & $1.62 \pm 0.01$ & $0.19 \pm 0.03$ & $2002-2010$ & $3.43 \pm 1.68$ & 36.1 \\
\hline (228502) $2001 \mathrm{TE} 2$ & 1.08 & 0.20 & 7.62 & $0.30 \pm 0.05$ & $0.19 \pm 0.06$ & 2000-2009 & $3.42 \pm 2.72$ & 21.2 \\
\hline 2003 TL4 & 0.78 & 0.38 & 12.15 & $0.38 \pm 0.07$ & $0.22 \pm 0.07$ & 2003-2009 & $3.39 \pm 2.83$ & 34.6 \\
\hline 2000 TJ1 & 1.16 & 0.08 & 39.54 & $0.25 \pm 0.04$ & $0.40 \pm 0.16$ & $2000-2010$ & $3.37 \pm 2.89$ & 18.9 \\
\hline 2000 YF29 & 1.49 & 0.37 & 6.30 & $0.24 \pm 0.04$ & $0.25 \pm 0.15$ & $2000-2011$ & $3.34 \pm 2.66$ & 12.8 \\
\hline 2010 OL101 & 2.61 & 0.60 & 26.07 & $0.20 \pm 0.02$ & $0.32 \pm 0.09$ & 132 days & $3.31 \pm 3.01$ & 5.5 \\
\hline 2008 EV5 & 0.96 & 0.08 & 7.44 & $0.40 \pm 0.01$ & $0.14 \pm 0.01$ & $2008-2010$ & $3.29 \pm 2.57$ & 24.6 \\
\hline 2010 ON101 & 1.63 & 0.41 & 9.31 & $0.24 \pm 0.02$ & $0.22 \pm 0.04$ & 2010-2012 & $3.29 \pm 2.61$ & 11.1 \\
\hline 2010 OD101 & 1.62 & 0.36 & 15.39 & $0.20 \pm 0.03$ & $0.22 \pm 0.07$ & 80 days & $3.29 \pm 2.48$ & 11.2 \\
\hline 2004 SB20 & 1.18 & 0.41 & 30.28 & $0.43 \pm 0.08$ & $0.41 \pm 0.12$ & 2004-2008 & $3.28 \pm 2.41$ & 17.9 \\
\hline (136874) 1998 FH74 & 2.20 & 0.88 & 21.26 & $3.40 \pm 0.13$ & $0.07 \pm 0.02$ & $1995-2011$ & $3.28 \pm 1.44$ & 7.0 \\
\hline 2007 BG29 & 0.83 & 0.33 & 18.51 & $0.65 \pm 0.02$ & $0.26 \pm 0.05$ & 2007-2012 & $3.26 \pm 2.22$ & 30.1 \\
\hline 2007 YQ56 & 1.14 & 0.29 & 26.46 & $0.34 \pm 0.07$ & $0.16 \pm 0.05$ & $2007-2008$ & $3.26 \pm 2.55$ & 18.7 \\
\hline 1996 GQ & 1.99 & 0.50 & 0.89 & $0.21 \pm 0.00$ & $0.02 \pm 0.00$ & $1996-2010$ & $3.25 \pm 2.64$ & 8.1 \\
\hline (285179) 1996 TY11 & 1.23 & 0.54 & 13.93 & $0.62 \pm 0.12$ & $0.09 \pm 0.03$ & $1996-2011$ & $3.24 \pm 2.24$ & 16.7 \\
\hline 2010 JN71 & 2.39 & 0.59 & 17.72 & $0.21 \pm 0.00$ & $0.11 \pm 0.02$ & 38 days & $3.22 \pm 2.29$ & 6.1 \\
\hline (85990) 1999 JV6 & 1.01 & 0.31 & 5.32 & $0.45 \pm 0.03$ & $0.10 \pm 0.02$ & $1999-2012$ & $3.18 \pm 2.33$ & 22.0 \\
\hline (138971) $2001 \mathrm{CB} 21$ & 1.03 & 0.33 & 7.90 & $0.58 \pm 0.11$ & $0.24 \pm 0.12$ & 2001-2011 & $3.13 \pm 2.48$ & 20.8 \\
\hline 2010 CN141 & 1.52 & 0.40 & 23.81 & $0.25 \pm 0.01$ & $0.03 \pm 0.01$ & 50 days & $3.12 \pm 2.21$ & 11.7 \\
\hline 2003 TJ2 & 1.32 & 0.47 & 17.44 & $0.45 \pm 0.09$ & $0.23 \pm 0.07$ & 2003-2012 & $3.11 \pm 2.62$ & 14.4 \\
\hline 2010 NZ1 & 1.37 & 0.65 & 32.79 & $0.85 \pm 0.05$ & $0.04 \pm 0.01$ & $1997-2010$ & $3.09 \pm 1.94$ & 13.5 \\
\hline
\end{tabular}


Table 4-Continued

\begin{tabular}{|c|c|c|c|c|c|c|c|c|}
\hline NEA & $\begin{array}{c}a \\
(\mathrm{AU})\end{array}$ & $e$ & $\begin{array}{c}i \\
(\operatorname{deg})\end{array}$ & $\begin{array}{l}D \\
\quad(\mathrm{~km})\end{array}$ & $p_{V}$ & Arc & $\begin{array}{c}d a / d t \\
10^{-4} \mathrm{AU} / \mathrm{Myr}\end{array}$ & $\begin{array}{c}\Delta \rho \\
(\mathrm{km})\end{array}$ \\
\hline (277617) $2006 \mathrm{BT} 7$ & 1.52 & 0.63 & 16.14 & $0.67 \pm 0.10$ & $0.19 \pm 0.08$ & 2006-2012 & $3.08 \pm 1.94$ & 11.5 \\
\hline $2010 \mathrm{CO} 1$ & 1.01 & 0.22 & 23.97 & $0.38 \pm 0.00$ & $0.03 \pm 0.00$ & 15 days & $3.07 \pm 2.16$ & 21.1 \\
\hline 2004 XK50 & 1.45 & 0.69 & 38.21 & $1.04 \pm 0.08$ & $0.04 \pm 0.01$ & $2004-2011$ & $3.05 \pm 1.83$ & 12.2 \\
\hline 2010 JH87 & 1.54 & 0.54 & 43.77 & $0.43 \pm 0.07$ & $0.13 \pm 0.06$ & $2010-2011$ & $3.05 \pm 2.32$ & 11.2 \\
\hline 2005 GY110 & 1.85 & 0.69 & 12.64 & $0.66 \pm 0.02$ & $0.19 \pm 0.03$ & $2005-2010$ & $2.98 \pm 1.84$ & 8.3 \\
\hline (154993) 2005 EA94 & 1.52 & 0.66 & 10.32 & $0.83 \pm 0.05$ & $0.20 \pm 0.05$ & $2002-2008$ & $2.94 \pm 1.88$ & 11.0 \\
\hline $2007 \mathrm{YZ}$ & 1.04 & 0.36 & 16.42 & $0.53 \pm 0.10$ & $0.09 \pm 0.04$ & $2007-2011$ & $2.93 \pm 2.23$ & 19.3 \\
\hline (85989) 1999 JD6 & 0.88 & 0.63 & 17.05 & $1.46 \pm 0.02$ & $0.13 \pm 0.03$ & $1999-2010$ & $2.93 \pm 1.63$ & 24.7 \\
\hline (164201) $2004 \mathrm{EC}$ & 2.00 & 0.86 & 34.64 & $2.87 \pm 0.57$ & $0.12 \pm 0.04$ & $2004-2010$ & $2.88 \pm 1.65$ & 7.1 \\
\hline (87024) 2000 JS66 & 1.20 & 0.19 & 14.43 & $0.31 \pm 0.06$ & $0.63 \pm 0.34$ & $2000-2009$ & $2.86 \pm 2.14$ & 15.3 \\
\hline (252558) $2001 \mathrm{WT} 1$ & 1.09 & 0.40 & 7.15 & $0.53 \pm 0.03$ & $0.06 \pm 0.01$ & $2001-2010$ & $2.84 \pm 2.00$ & 17.5 \\
\hline (162483) 2000 PJ5 & 0.87 & 0.37 & 51.18 & $0.92 \pm 0.01$ & $0.23 \pm 0.03$ & $2000-2010$ & $2.84 \pm 1.61$ & 24.3 \\
\hline 2010 LU134 & 1.90 & 0.55 & 27.38 & $0.32 \pm 0.03$ & $0.44 \pm 0.11$ & 138 days & $2.81 \pm 1.85$ & 7.5 \\
\hline (38086) Beowulf & 1.42 & 0.57 & 23.67 & $0.70 \pm 0.20$ & $0.37 \pm 0.12$ & $1992-2011$ & $2.79 \pm 3.43$ & 11.5 \\
\hline 2010 OC103 & 1.19 & 0.67 & 23.11 & $1.47 \pm 0.31$ & $0.02 \pm 0.01$ & $2006-2011$ & $2.78 \pm 1.60$ & 14.9 \\
\hline 2010 DJ56 & 1.25 & 0.25 & 34.84 & $0.32 \pm 0.05$ & $0.33 \pm 0.15$ & $2003-2010$ & $2.76 \pm 2.30$ & 13.8 \\
\hline $2010 \mathrm{JF} 88$ & 2.23 & 0.66 & 17.45 & $0.45 \pm 0.01$ & $0.32 \pm 0.07$ & $2010-2011$ & $2.74 \pm 2.25$ & 5.8 \\
\hline (162416) 2000 EH26 & 1.85 & 0.48 & 0.40 & $0.14 \pm 0.03$ & $0.18 \pm 0.14$ & $2000-2005$ & $2.70 \pm 3.05$ & 7.5 \\
\hline 2007 MK13 & 1.02 & 0.14 & 19.88 & $0.39 \pm 0.01$ & $0.12 \pm 0.02$ & $2007-2011$ & $2.69 \pm 2.10$ & 18.1 \\
\hline $2006 \mathrm{LF}$ & 2.14 & 0.66 & 7.97 & $0.50 \pm 0.10$ & $0.25 \pm 0.08$ & 2002-2009 & $2.66 \pm 1.97$ & 5.9 \\
\hline 2008 WB59 & 1.04 & 0.19 & 25.65 & $0.41 \pm 0.07$ & $0.20 \pm 0.10$ & 2008-2010 & $2.63 \pm 1.97$ & 17.2 \\
\hline 2010 JA35 & 2.16 & 0.58 & 29.00 & $0.31 \pm 0.05$ & $0.11 \pm 0.06$ & 201 days & $2.62 \pm 2.22$ & 5.8 \\
\hline 2005 EJ & 1.45 & 0.15 & 12.46 & $0.23 \pm 0.04$ & $0.43 \pm 0.24$ & 2005-2012 & $2.57 \pm 2.31$ & 10.4 \\
\hline 2010 FO92 & 2.14 & 0.52 & 6.75 & $0.25 \pm 0.01$ & $0.28 \pm 0.05$ & 238 days & $2.55 \pm 2.05$ & 5.7 \\
\hline 2010 CN44 & 2.85 & 0.68 & 3.84 & $0.34 \pm 0.01$ & $0.19 \pm 0.04$ & 115 days & $2.55 \pm 1.93$ & 3.7 \\
\hline 2009 UX17 & 1.19 & 0.08 & 10.80 & $0.31 \pm 0.01$ & $0.04 \pm 0.01$ & 134 days & $2.54 \pm 2.19$ & 13.7 \\
\hline 2010 GV147 & 0.96 & 0.66 & 44.05 & $2.05 \pm 0.04$ & $0.02 \pm 0.00$ & $2010-2011$ & $2.51 \pm 1.24$ & 18.7 \\
\hline $2010 \mathrm{FQ}$ & 1.53 & 0.36 & 10.55 & $0.29 \pm 0.00$ & $0.21 \pm 0.03$ & 2010-2012 & $2.50 \pm 1.81$ & 9.3 \\
\hline (138947) 2001 BA40 & 1.12 & 0.25 & 12.85 & $0.44 \pm 0.09$ & $0.42 \pm 0.26$ & $2001-2007$ & $2.48 \pm 2.01$ & 14.7 \\
\hline (207945) $1991 \mathrm{JW}$ & 1.04 & 0.12 & 8.71 & $0.42 \pm 0.03$ & $0.16 \pm 0.04$ & $1955-2009$ & $2.45 \pm 1.89$ & 16.2 \\
\hline
\end{tabular}


Table 4-Continued

\begin{tabular}{|c|c|c|c|c|c|c|c|c|}
\hline NEA & $\begin{array}{c}a \\
(\mathrm{AU})\end{array}$ & $e$ & $\begin{array}{c}i \\
(\operatorname{deg})\end{array}$ & $\begin{array}{l}D \\
\quad(\mathrm{~km})\end{array}$ & $p_{V}$ & Arc & $\begin{array}{c}d a / d t \\
10^{-4} \mathrm{AU} / \mathrm{Myr}\end{array}$ & $\begin{array}{c}\Delta \rho \\
(\mathrm{km})\end{array}$ \\
\hline 2010 DM21 & 2.86 & 0.66 & 21.14 & $0.30 \pm 0.01$ & $0.13 \pm 0.02$ & 48 days & $2.44 \pm 1.84$ & 3.5 \\
\hline $2006 \mathrm{KD} 1$ & 2.48 & 0.78 & 30.71 & $1.14 \pm 0.12$ & $0.18 \pm 0.05$ & 2006-2010 & $2.41 \pm 1.67$ & 4.3 \\
\hline (221980) 1996 EO & 1.34 & 0.40 & 21.60 & $0.43 \pm 0.05$ & $0.20 \pm 0.05$ & 1996-2009 & $2.40 \pm 1.85$ & 10.8 \\
\hline (52381) $1993 \mathrm{HA}$ & 1.28 & 0.14 & 7.73 & $0.34 \pm 0.10$ & $0.14 \pm 0.11$ & 1993-2009 & $2.39 \pm 2.83$ & 11.6 \\
\hline 1998 SB15 & 1.23 & 0.16 & 15.63 & $0.34 \pm 0.03$ & $0.06 \pm 0.01$ & $1998-2010$ & $2.36 \pm 1.95$ & 12.1 \\
\hline 2010 HZ108 & 1.25 & 0.21 & 22.88 & $0.36 \pm 0.07$ & $0.06 \pm 0.03$ & 180 days & $2.32 \pm 1.93$ & 11.7 \\
\hline (99942) Apophis & 0.92 & 0.19 & 3.33 & $0.27 \pm 0.06$ & $0.33 \pm 0.08$ & 2004-2012 & $2.32 \pm 2.29$ & 18.3 \\
\hline (239849) 1999 VO11 & 2.25 & 0.64 & 15.80 & $0.45 \pm 0.06$ & $0.32 \pm 0.13$ & $1999-2010$ & $2.31 \pm 1.76$ & 4.8 \\
\hline 2003 WD158 & 1.43 & 0.41 & 16.71 & $0.44 \pm 0.09$ & $0.29 \pm 0.09$ & $2003-2008$ & $2.30 \pm 1.94$ & 9.5 \\
\hline (238456) 2004 RK & 1.39 & 0.30 & 18.15 & $0.33 \pm 0.04$ & $0.31 \pm 0.14$ & $1986-2010$ & $2.30 \pm 1.87$ & 9.8 \\
\hline (275558) 1999 RH33 & 1.55 & 0.17 & 11.02 & $0.23 \pm 0.04$ & $0.73 \pm 0.35$ & $1999-2012$ & $2.27 \pm 1.89$ & 8.2 \\
\hline (55408) 2001 TC2 & 1.10 & 0.22 & 30.39 & $0.46 \pm 0.01$ & $0.34 \pm 0.05$ & $1979-2010$ & $2.27 \pm 1.60$ & 13.8 \\
\hline 2009 SO103 & 2.00 & 0.66 & 29.46 & $0.67 \pm 0.01$ & $0.43 \pm 0.07$ & 2006-2010 & $2.27 \pm 1.56$ & 5.6 \\
\hline 2009 UK & 1.98 & 0.48 & 3.53 & $0.28 \pm 0.02$ & $0.22 \pm 0.05$ & $1984-2010$ & $2.25 \pm 1.74$ & 5.6 \\
\hline (3200) Phaethon & 1.27 & 0.89 & 22.23 & $11.00 \pm 0.40$ & $0.02 \pm 0.01$ & $1983-2012$ & $2.21 \pm 0.74$ & 10.8 \\
\hline 2007 FE1 & 2.02 & 0.55 & 9.85 & $0.39 \pm 0.01$ & $0.35 \pm 0.07$ & $2007-2010$ & $2.21 \pm 1.89$ & 5.4 \\
\hline (85938) 1999 DJ4 & 1.85 & 0.48 & 9.15 & $0.48 \pm 0.10$ & $0.28 \pm 0.23$ & 1999-2009 & $2.18 \pm 1.86$ & 6.0 \\
\hline (163132) 2002 CU11 & 1.22 & 0.30 & 48.77 & $0.46 \pm 0.02$ & $0.41 \pm 0.06$ & $2002-2010$ & $2.17 \pm 1.70$ & 11.3 \\
\hline (140158) 2001 SX169 & 1.35 & 0.46 & 2.51 & $0.57 \pm 0.01$ & $0.29 \pm 0.06$ & 2001-2009 & $2.15 \pm 1.59$ & 9.6 \\
\hline (277039) 2005 CF41 & 1.65 & 0.59 & 15.89 & $0.68 \pm 0.02$ & $0.16 \pm 0.03$ & 2005-2011 & $2.12 \pm 1.49$ & 7.0 \\
\hline 2010 EX119 & 1.90 & 0.60 & 15.57 & $0.56 \pm 0.01$ & $0.08 \pm 0.01$ & $2010-2010$ & $2.10 \pm 1.58$ & 5.6 \\
\hline 2010 FB81 & 2.58 & 0.60 & 9.48 & $0.32 \pm 0.01$ & $0.05 \pm 0.01$ & 118 days & $2.10 \pm 1.69$ & 3.5 \\
\hline $2009 \mathrm{VO} 24$ & 1.55 & 0.46 & 6.05 & $0.47 \pm 0.02$ & $0.10 \pm 0.02$ & 2009-2011 & $2.08 \pm 1.73$ & 7.6 \\
\hline 2010 JG88 & 1.37 & 0.38 & 30.61 & $0.46 \pm 0.01$ & $0.14 \pm 0.02$ & $2010-2010$ & $2.08 \pm 1.60$ & 9.0 \\
\hline 2010 GE25 & 2.07 & 0.47 & 21.65 & $0.30 \pm 0.05$ & $0.23 \pm 0.10$ & 40 days & $2.05 \pm 1.84$ & 4.8 \\
\hline $2010 \mathrm{JN} 33$ & 1.72 & 0.34 & 55.30 & $0.29 \pm 0.01$ & $0.09 \pm 0.02$ & 4 days & $2.05 \pm 1.74$ & 6.4 \\
\hline (262623) 2006 WY2 & 0.98 & 0.33 & 27.55 & $0.76 \pm 0.08$ & $0.12 \pm 0.04$ & 2006-2012 & $2.05 \pm 1.55$ & 14.7 \\
\hline 2006 OD7 & 1.33 & 0.17 & 30.33 & $0.33 \pm 0.07$ & $0.27 \pm 0.08$ & 2006-2009 & $2.04 \pm 1.71$ & 9.3 \\
\hline (5604) $1992 \mathrm{FE}$ & 0.93 & 0.41 & 4.79 & $0.96 \pm 0.01$ & $0.52 \pm 0.08$ & $1985-2010$ & $1.99 \pm 1.33$ & 15.6 \\
\hline 2007 JZ20 & 1.31 & 0.34 & 40.48 & $0.48 \pm 0.04$ & $0.31 \pm 0.07$ & 2004-2010 & $1.97 \pm 1.47$ & 9.2 \\
\hline
\end{tabular}


Table 4-Continued

\begin{tabular}{|c|c|c|c|c|c|c|c|c|}
\hline NEA & $\begin{array}{c}a \\
(\mathrm{AU})\end{array}$ & $e$ & $\begin{array}{c}i \\
(\mathrm{deg})\end{array}$ & $\begin{array}{l}D \\
\qquad(\mathrm{~km})\end{array}$ & $p_{V}$ & Arc & $\begin{array}{c}d a / d t \\
10^{-4} \mathrm{AU} / \mathrm{Myr}\end{array}$ & $\begin{array}{c}\Delta \rho \\
(\mathrm{km})\end{array}$ \\
\hline 1998 OK1 & 1.36 & 0.43 & 13.99 & $0.56 \pm 0.01$ & $0.10 \pm 0.02$ & $1998-2010$ & $1.96 \pm 1.38$ & 8.7 \\
\hline $2010 \mathrm{AE}$ & 1.49 & 0.38 & 15.97 & $0.41 \pm 0.00$ & $0.18 \pm 0.04$ & $2010-2010$ & $1.96 \pm 1.74$ & 7.6 \\
\hline$(218017) 2001$ XV266 & 1.20 & 0.19 & 12.00 & $0.45 \pm 0.01$ & $0.12 \pm 0.03$ & $2001-2010$ & $1.95 \pm 1.66$ & 10.4 \\
\hline (302591) 2002 QE7 & 1.47 & 0.18 & 12.11 & $0.32 \pm 0.06$ & $0.34 \pm 0.20$ & $2002-2011$ & $1.95 \pm 1.93$ & 7.7 \\
\hline $2000 \mathrm{CO} 101$ & 1.08 & 0.09 & 15.32 & $0.53 \pm 0.16$ & $0.11 \pm 0.19$ & $2000-2011$ & $1.93 \pm 1.61$ & 12.1 \\
\hline $2010 \mathrm{JE}$ & 2.78 & 0.66 & 13.90 & $0.41 \pm 0.05$ & $0.09 \pm 0.03$ & 194 days & $1.93 \pm 1.46$ & 2.9 \\
\hline (238063) 2003 EG & 1.74 & 0.71 & 31.75 & $1.47 \pm 0.30$ & $0.23 \pm 0.13$ & $1996-2010$ & $1.92 \pm 1.24$ & 5.9 \\
\hline 2005 ED318 & 1.85 & 0.45 & 2.39 & $0.20 \pm 0.01$ & $0.21 \pm 0.04$ & 2005-2010 & $1.87 \pm 1.77$ & 5.2 \\
\hline 2010 FC81 & 2.67 & 0.63 & 1.68 & $0.40 \pm 0.01$ & $0.02 \pm 0.00$ & 97 days & $1.87 \pm 1.71$ & 3.0 \\
\hline (65679) 1989 UQ & 0.92 & 0.26 & 1.29 & $0.73 \pm 0.18$ & $0.06 \pm 0.06$ & $1954-2011$ & $1.85 \pm 1.59$ & 14.8 \\
\hline $2010 \mathrm{~KB} 61$ & 1.27 & 0.23 & 44.60 & $0.42 \pm 0.01$ & $0.06 \pm 0.01$ & 189 days & $1.85 \pm 1.51$ & 9.0 \\
\hline (138911) $2001 \mathrm{AE} 2$ & 1.35 & 0.08 & 1.66 & $0.34 \pm 0.07$ & $0.34 \pm 0.22$ & 1984-2012 & $1.83 \pm 1.61$ & 8.2 \\
\hline 1998 SE36 & 1.34 & 0.10 & 11.68 & $0.34 \pm 0.07$ & $0.30 \pm 0.22$ & 1998-2009 & $1.83 \pm 1.40$ & 8.2 \\
\hline $2009 \mathrm{XD}$ & 2.45 & 0.67 & 31.47 & $0.62 \pm 0.02$ & $0.15 \pm 0.03$ & 2009-2010 & $1.83 \pm 1.30$ & 3.3 \\
\hline 2010 MF1 & 2.50 & 0.59 & 9.12 & $0.36 \pm 0.00$ & $0.18 \pm 0.03$ & 177 days & $1.82 \pm 1.35$ & 3.2 \\
\hline (152889) 2000 CF59 & 1.68 & 0.64 & 41.59 & $1.02 \pm 0.02$ & $0.39 \pm 0.07$ & $1998-2010$ & $1.79 \pm 1.26$ & 5.8 \\
\hline (154035) 2002 CV59 & 1.21 & 0.53 & 49.06 & $1.10 \pm 0.12$ & $0.15 \pm 0.03$ & $2002-2010$ & $1.79 \pm 1.26$ & 9.4 \\
\hline (152978) 2000 GJ147 & 1.16 & 0.24 & 25.01 & $0.53 \pm 0.01$ & $0.11 \pm 0.02$ & $2000-2010$ & $1.79 \pm 1.40$ & 10.0 \\
\hline 2010 DW1 & 1.22 & 0.20 & 23.77 & $0.45 \pm 0.00$ & $0.09 \pm 0.01$ & $2002-2010$ & $1.78 \pm 1.36$ & 9.2 \\
\hline (138937) 2001 BK16 & 2.07 & 0.68 & 31.83 & $0.92 \pm 0.20$ & $0.21 \pm 0.06$ & $1998-2010$ & $1.78 \pm 1.30$ & 4.2 \\
\hline 2010 HR80 & 1.35 & 0.50 & 26.71 & $0.78 \pm 0.01$ & $0.02 \pm 0.00$ & 2010-2010 & $1.77 \pm 1.21$ & 7.9 \\
\hline 2010 MR87 & 1.73 & 0.39 & 34.98 & $0.36 \pm 0.06$ & $0.22 \pm 0.10$ & 2003-2010 & $1.76 \pm 1.36$ & 5.4 \\
\hline 2009 WC26 & 2.17 & 0.70 & 12.02 & $0.92 \pm 0.01$ & $0.23 \pm 0.04$ & $2007-2010$ & $1.76 \pm 1.23$ & 3.8 \\
\hline 2003 SL5 & 2.11 & 0.47 & 6.10 & $0.34 \pm 0.07$ & $0.38 \pm 0.22$ & 2003-2009 & $1.75 \pm 1.72$ & 4.0 \\
\hline 2010 JM151 & 1.70 & 0.48 & 16.64 & $0.54 \pm 0.02$ & $0.09 \pm 0.02$ & 2010-2012 & $1.68 \pm 1.30$ & 5.3 \\
\hline 2010 MU112 & 1.76 & 0.54 & 48.02 & $0.60 \pm 0.02$ & $0.03 \pm 0.01$ & 134 days & $1.68 \pm 1.23$ & 5.0 \\
\hline 2010 GZ6 & 1.40 & 0.14 & 44.84 & $0.37 \pm 0.06$ & $0.21 \pm 0.07$ & $2002-2010$ & $1.67 \pm 1.55$ & 7.0 \\
\hline 2010 NT1 & 1.46 & 0.22 & 39.52 & $0.38 \pm 0.06$ & $0.21 \pm 0.08$ & 99 days & $1.67 \pm 1.65$ & 6.6 \\
\hline (105141) 2000 NF11 & 1.42 & 0.19 & 14.82 & $0.35 \pm 0.05$ & $0.43 \pm 0.14$ & $1978-2005$ & $1.67 \pm 1.20$ & 6.9 \\
\hline (9162) Kwiila & 1.50 & 0.60 & 9.02 & $1.13 \pm 0.23$ & $0.09 \pm 0.04$ & 1987-2009 & $1.66 \pm 1.28$ & 6.4 \\
\hline
\end{tabular}


Table 4-Continued

\begin{tabular}{|c|c|c|c|c|c|c|c|c|}
\hline NEA & $\begin{array}{c}a \\
(\mathrm{AU})\end{array}$ & $e$ & $\begin{array}{c}i \\
(\operatorname{deg})\end{array}$ & $\begin{array}{l}D \\
\qquad(\mathrm{~km})\end{array}$ & $p_{V}$ & Arc & $\begin{array}{c}d a / d t \\
10^{-4} \mathrm{AU} / \mathrm{Myr}\end{array}$ & $\begin{array}{c}\Delta \rho \\
(\mathrm{km})\end{array}$ \\
\hline $2010 \mathrm{FZ} 80$ & 2.78 & 0.74 & 25.72 & $0.87 \pm 0.01$ & $0.02 \pm 0.00$ & $2010-2010$ & $1.65 \pm 1.14$ & 2.5 \\
\hline 2010 LF64 & 1.34 & 0.16 & 18.25 & $0.35 \pm 0.01$ & $0.03 \pm 0.01$ & 2 days & $1.65 \pm 1.40$ & 7.4 \\
\hline 2010 CP140 & 1.90 & 0.54 & 14.47 & $0.56 \pm 0.10$ & $0.11 \pm 0.03$ & 127 days & $1.62 \pm 1.19$ & 4.3 \\
\hline 2010 LT108 & 1.35 & 0.37 & 31.87 & $0.60 \pm 0.01$ & $0.06 \pm 0.01$ & 90 days & $1.59 \pm 1.33$ & 7.1 \\
\hline (22099) 2000 EX106 & 1.10 & 0.28 & 9.84 & $0.62 \pm 0.11$ & $0.29 \pm 0.16$ & $1994-2008$ & $1.58 \pm 1.17$ & 9.5 \\
\hline 2003 QH5 & 1.26 & 0.22 & 17.61 & $0.54 \pm 0.09$ & $0.06 \pm 0.03$ & $2003-2010$ & $1.58 \pm 1.38$ & 7.8 \\
\hline 2009 ST103 & 2.69 & 0.72 & 15.94 & $0.85 \pm 0.01$ & $0.14 \pm 0.02$ & $2000-2010$ & $1.57 \pm 1.17$ & 2.5 \\
\hline 1999 TX2 & 1.28 & 0.46 & 61.39 & $0.96 \pm 0.19$ & $0.11 \pm 0.03$ & $1999-2005$ & $1.57 \pm 1.36$ & 7.6 \\
\hline (52750) 1998 KK17 & 1.43 & 0.52 & 11.16 & $1.06 \pm 0.01$ & $0.39 \pm 0.08$ & 1992-2009 & $1.57 \pm 1.01$ & 6.4 \\
\hline 2010 GU21 & 2.18 & 0.56 & 3.19 & $0.51 \pm 0.04$ & $0.02 \pm 0.01$ & 136 days & $1.56 \pm 1.20$ & 3.4 \\
\hline (90373) 2003 SZ219 & 1.63 & 0.20 & 9.87 & $0.31 \pm 0.05$ & $0.58 \pm 0.30$ & $1998-2007$ & $1.56 \pm 1.28$ & 5.3 \\
\hline (172974) 2005 YW55 & 1.64 & 0.25 & 8.47 & $0.34 \pm 0.08$ & $0.30 \pm 0.24$ & $2002-2011$ & $1.55 \pm 1.46$ & 5.2 \\
\hline (280136) 2002 OM4 & 1.50 & 0.56 & 55.32 & $1.03 \pm 0.20$ & $0.30 \pm 0.09$ & $2002-2011$ & $1.55 \pm 1.18$ & 5.9 \\
\hline 1993 RA & 1.92 & 0.42 & 5.61 & $0.36 \pm 0.06$ & $0.40 \pm 0.23$ & $1993-2010$ & $1.54 \pm 1.25$ & 4.1 \\
\hline (10302) $1989 \mathrm{ML}$ & 1.27 & 0.14 & 4.38 & $0.24 \pm 0.04$ & $0.49 \pm 0.28$ & $1989-2006$ & $1.53 \pm 1.37$ & 7.5 \\
\hline 2010 FJ81 & 3.59 & 0.69 & 42.26 & $0.42 \pm 0.01$ & $0.05 \pm 0.01$ & 174 days & $1.52 \pm 1.31$ & 1.6 \\
\hline 2010 FY80 & 2.69 & 0.61 & 18.86 & $0.45 \pm 0.01$ & $0.12 \pm 0.02$ & 128 days & $1.52 \pm 1.17$ & 2.4 \\
\hline (25143) Itokawa & 1.32 & 0.28 & 1.62 & $0.45 \pm 0.18$ & $0.41 \pm 0.20$ & $1998-2010$ & $1.50 \pm 2.29$ & 6.9 \\
\hline 2000 GV147 & 1.75 & 0.46 & 10.57 & $0.50 \pm 0.10$ & $0.19 \pm 0.12$ & $2000-2009$ & $1.50 \pm 1.15$ & 4.6 \\
\hline 2010 CN1 & 1.50 & 0.44 & 20.97 & $0.64 \pm 0.06$ & $0.12 \pm 0.04$ & $2010-2011$ & $1.49 \pm 1.22$ & 5.7 \\
\hline 2010 DH77 & 3.27 & 0.71 & 34.37 & $0.58 \pm 0.01$ & $0.01 \pm 0.00$ & 107 days & $1.49 \pm 1.18$ & 1.8 \\
\hline (226198) 2002 UN3 & 1.74 & 0.26 & 8.70 & $0.31 \pm 0.05$ & $0.67 \pm 0.35$ & 1994-2009 & $1.49 \pm 1.30$ & 4.5 \\
\hline (263976) 2009 KD5 & 1.05 & 0.26 & 13.47 & $0.78 \pm 0.01$ & $0.13 \pm 0.02$ & $1950-2012$ & $1.47 \pm 1.04$ & 9.6 \\
\hline (164121) $2003 \mathrm{YT} 1$ & 1.11 & 0.29 & 44.06 & $1.10 \pm 0.08$ & $0.49 \pm 0.04$ & $1982-2011$ & $1.46 \pm 1.04$ & 8.8 \\
\hline 2001 HA4 & 2.68 & 0.80 & 17.20 & $1.85 \pm 0.04$ & $0.05 \pm 0.01$ & $2001-2010$ & $1.44 \pm 0.99$ & 2.3 \\
\hline (215442) 2002 MQ3 & 0.91 & 0.27 & 36.28 & $1.05 \pm 0.02$ & $0.08 \pm 0.00$ & $2002-2009$ & $1.44 \pm 0.91$ & 11.6 \\
\hline (42286) 2001 TN41 & 1.42 & 0.39 & 24.07 & $0.70 \pm 0.20$ & $1.00 \pm 0.30$ & $1975-2012$ & $1.43 \pm 1.23$ & 5.9 \\
\hline 2010 CH18 & 2.61 & 0.57 & 27.15 & $0.39 \pm 0.01$ & $0.26 \pm 0.04$ & 128 days & $1.42 \pm 1.17$ & 2.4 \\
\hline $2002 \mathrm{YF} 4$ & 1.75 & 0.33 & 33.68 & $0.38 \pm 0.01$ & $0.43 \pm 0.05$ & $2002-2010$ & $1.41 \pm 1.07$ & 4.2 \\
\hline 2004 JX20 & 0.90 & 0.27 & 10.52 & $1.13 \pm 0.01$ & $0.02 \pm 0.00$ & 2004-2011 & $1.40 \pm 1.02$ & 11.4 \\
\hline
\end{tabular}


Table 4-Continued

\begin{tabular}{|c|c|c|c|c|c|c|c|c|}
\hline NEA & $\begin{array}{c}a \\
(\mathrm{AU})\end{array}$ & $e$ & $\begin{array}{c}i \\
(\operatorname{deg})\end{array}$ & $\begin{array}{l}D \\
\qquad(\mathrm{~km})\end{array}$ & $p_{V}$ & Arc & $\begin{array}{c}d a / d t \\
10^{-4} \mathrm{AU} / \mathrm{Myr}\end{array}$ & $\begin{array}{l}\Delta \rho \\
(\mathrm{km})\end{array}$ \\
\hline (153220) 2000 YN29 & 2.53 & 0.68 & 5.87 & $0.81 \pm 0.16$ & $0.27 \pm 0.09$ & $1997-2009$ & $1.40 \pm 1.09$ & 2.4 \\
\hline (152931) 2000 EA107 & 0.93 & 0.46 & 28.58 & $1.65 \pm 0.04$ & $0.24 \pm 0.04$ & $2000-2011$ & $1.39 \pm 0.86$ & 10.9 \\
\hline 2005 XD1 & 1.62 & 0.31 & 18.29 & $0.41 \pm 0.01$ & $0.18 \pm 0.03$ & $2005-2011$ & $1.39 \pm 1.13$ & 4.7 \\
\hline (220124) 2002 TE66 & 1.46 & 0.38 & 51.34 & $0.61 \pm 0.03$ & $0.21 \pm 0.04$ & $2002-2009$ & $1.37 \pm 1.01$ & 5.4 \\
\hline (99799) 2002 LJ3 & 1.46 & 0.28 & 7.56 & $0.49 \pm 0.10$ & $0.42 \pm 0.25$ & $1989-2012$ & $1.34 \pm 1.09$ & 5.3 \\
\hline 2009 UP1 & 1.52 & 0.40 & 17.28 & $0.61 \pm 0.02$ & $0.04 \pm 0.01$ & 95 days & $1.34 \pm 0.94$ & 5.0 \\
\hline (242147) 2003 BH84 & 1.96 & 0.72 & 23.40 & $1.69 \pm 0.01$ & $0.11 \pm 0.03$ & 2003-2010 & $1.33 \pm 0.88$ & 3.4 \\
\hline 2009 SX1 & 1.72 & 0.45 & 8.29 & $0.56 \pm 0.01$ & $0.17 \pm 0.03$ & $2002-2010$ & $1.32 \pm 1.09$ & 4.1 \\
\hline 2010 GH65 & 2.71 & 0.61 & 21.08 & $0.49 \pm 0.02$ & $0.24 \pm 0.06$ & $2001-2010$ & $1.31 \pm 1.18$ & 2.1 \\
\hline (237551) 2000 WQ19 & 1.41 & 0.35 & 34.28 & $0.63 \pm 0.04$ & $0.23 \pm 0.05$ & $2000-2010$ & $1.30 \pm 0.97$ & 5.5 \\
\hline (306462) 1999 RC32 & 1.84 & 0.43 & 30.97 & $0.48 \pm 0.06$ & $0.38 \pm 0.09$ & $1999-2010$ & $1.30 \pm 1.12$ & 3.7 \\
\hline $2010 \mathrm{BH} 2$ & 2.35 & 0.46 & 24.62 & $0.36 \pm 0.07$ & $0.21 \pm 0.10$ & 149 days & $1.29 \pm 1.23$ & 2.5 \\
\hline (6489) Golevka & 2.50 & 0.61 & 2.28 & $0.53 \pm 0.03$ & $0.15 \pm 0.02$ & $1991-2011$ & $1.25 \pm 1.14$ & 2.2 \\
\hline (9202) 1993 PB & 1.42 & 0.61 & 40.82 & $1.62 \pm 0.05$ & $0.27 \pm 0.06$ & 1993-2009 & $1.25 \pm 0.78$ & 5.1 \\
\hline 2010 HQ80 & 1.57 & 0.49 & 27.86 & $0.89 \pm 0.15$ & $0.02 \pm 0.01$ & $2010-2010$ & $1.25 \pm 1.11$ & 4.4 \\
\hline (55532) 2001 WG2 & 1.79 & 0.70 & 38.50 & $1.96 \pm 0.40$ & $0.14 \pm 0.04$ & $1953-2006$ & $1.24 \pm 0.95$ & 3.6 \\
\hline 2010 LR33 & 1.69 & 0.46 & 5.83 & $0.66 \pm 0.01$ & $0.21 \pm 0.04$ & $2001-2012$ & $1.23 \pm 1.04$ & 3.9 \\
\hline 2008 EB9 & 1.56 & 0.22 & 21.35 & $0.43 \pm 0.08$ & $0.07 \pm 0.04$ & $2008-2010$ & $1.23 \pm 0.96$ & 4.4 \\
\hline (4660) Nereus & 1.49 & 0.36 & 1.43 & $0.40 \pm 0.10$ & $0.55 \pm 0.17$ & $1981-2010$ & $1.23 \pm 1.37$ & 4.7 \\
\hline (161999) $1989 \mathrm{RC}$ & 2.31 & 0.52 & 7.39 & $0.46 \pm 0.02$ & $0.21 \pm 0.04$ & $1989-2010$ & $1.20 \pm 0.91$ & 2.4 \\
\hline (152964) 2000 GP82 & 1.40 & 0.39 & 13.22 & $0.79 \pm 0.15$ & $0.34 \pm 0.18$ & $1995-2010$ & $1.20 \pm 0.88$ & 5.1 \\
\hline $2002 \mathrm{WP}$ & 1.45 & 0.22 & 19.15 & $0.52 \pm 0.08$ & $0.32 \pm 0.13$ & $2002-2010$ & $1.19 \pm 1.06$ & 4.8 \\
\hline 2010 GX62 & 2.95 & 0.70 & 21.64 & $0.79 \pm 0.01$ & $0.01 \pm 0.00$ & 180 days & $1.17 \pm 0.86$ & 1.6 \\
\hline 2006 EE1 & 1.20 & 0.28 & 36.40 & $0.78 \pm 0.02$ & $0.04 \pm 0.01$ & $2006-2010$ & $1.17 \pm 0.80$ & 6.2 \\
\hline 2009 AV & 1.03 & 0.07 & 45.87 & $0.87 \pm 0.02$ & $0.15 \pm 0.03$ & 2009-2012 & $1.16 \pm 0.90$ & 7.7 \\
\hline (138359) 2000 GX127 & 1.14 & 0.36 & 20.24 & $1.08 \pm 0.03$ & $0.09 \pm 0.02$ & $2000-2010$ & $1.15 \pm 0.84$ & 6.6 \\
\hline $2009 \mathrm{SP}$ & 2.22 & 0.49 & 25.28 & $0.48 \pm 0.10$ & $0.12 \pm 0.07$ & 168 days & $1.15 \pm 1.03$ & 2.4 \\
\hline (159686) 2002 LB6 & 1.80 & 0.69 & 24.69 & $1.84 \pm 0.01$ & $0.17 \pm 0.03$ & $1997-2010$ & $1.13 \pm 0.68$ & 3.3 \\
\hline 2010 CG18 & 1.44 & 0.23 & 10.16 & $0.56 \pm 0.02$ & $0.02 \pm 0.00$ & $2010-2011$ & $1.12 \pm 0.94$ & 4.6 \\
\hline 2007 WV4 & 1.49 & 0.44 & 38.34 & $0.89 \pm 0.16$ & $0.04 \pm 0.02$ & $2007-2010$ & $1.11 \pm 0.85$ & 4.3 \\
\hline
\end{tabular}


Table 4-Continued

\begin{tabular}{|c|c|c|c|c|c|c|c|c|}
\hline NEA & $\begin{array}{c}a \\
(\mathrm{AU})\end{array}$ & $e$ & $\begin{array}{c}i \\
(\operatorname{deg})\end{array}$ & $\begin{array}{l}D \\
\quad(\mathrm{~km})\end{array}$ & $p_{V}$ & Arc & $\begin{array}{c}d a / d t \\
10^{-4} \mathrm{AU} / \mathrm{Myr}\end{array}$ & $\begin{array}{c}\Delta \rho \\
(\mathrm{km})\end{array}$ \\
\hline 2005 JA22 & 1.53 & 0.30 & 13.24 & $0.67 \pm 0.17$ & $0.16 \pm 0.12$ & 2005-2012 & $1.10 \pm 1.07$ & 4.0 \\
\hline 2010 DM56 & 1.31 & 0.29 & 25.61 & $0.77 \pm 0.00$ & $0.02 \pm 0.00$ & 121 days & $1.09 \pm 0.84$ & 5.1 \\
\hline (35107) $1991 \mathrm{VH}$ & 1.14 & 0.14 & 13.91 & $1.12 \pm 0.23$ & $0.27 \pm 0.20$ & $1991-2008$ & $1.08 \pm 0.92$ & 6.2 \\
\hline 2002 NW16 & 1.11 & 0.03 & 14.16 & $0.85 \pm 0.01$ & $0.16 \pm 0.03$ & $2002-2011$ & $1.05 \pm 0.87$ & 6.3 \\
\hline (277616) $2006 \mathrm{BN} 6$ & 2.56 & 0.71 & 18.38 & $1.24 \pm 0.01$ & $0.13 \pm 0.03$ & $2002-2010$ & $1.04 \pm 0.74$ & 1.8 \\
\hline (137805) 1999 YK5 & 0.83 & 0.56 & 16.74 & $3.88 \pm 0.16$ & $0.03 \pm 0.01$ & 1999-2012 & $1.03 \pm 0.57$ & 9.5 \\
\hline (161989) Cacus & 1.12 & 0.21 & 26.06 & $1.13 \pm 0.07$ & $0.20 \pm 0.05$ & $1978-2010$ & $1.02 \pm 0.85$ & 6.0 \\
\hline 2010 CD19 & 2.26 & 0.58 & 20.70 & $0.74 \pm 0.01$ & $0.25 \pm 0.05$ & $2009-2010$ & $1.02 \pm 0.84$ & 2.1 \\
\hline (3671) Dionysus & 2.20 & 0.54 & 13.55 & $0.89 \pm 0.11$ & $0.67 \pm 0.37$ & $1984-2011$ & $1.02 \pm 0.80$ & 2.2 \\
\hline 2002 NP1 & 1.25 & 0.17 & 19.12 & $0.81 \pm 0.16$ & $0.25 \pm 0.08$ & $2002-2010$ & $1.02 \pm 0.91$ & 5.1 \\
\hline (1865) Cerberus & 1.08 & 0.47 & 16.10 & $1.61 \pm 0.01$ & $0.14 \pm 0.02$ & $1971-2008$ & $1.00 \pm 0.68$ & 6.3 \\
\hline (4197) $1982 \mathrm{TA}$ & 2.30 & 0.77 & 12.57 & $3.04 \pm 0.16$ & $0.28 \pm 0.08$ & $1954-2010$ & $1.00 \pm 0.62$ & 2.0 \\
\hline 2007 DK8 & 1.65 & 0.42 & 32.57 & $0.79 \pm 0.16$ & $0.08 \pm 0.03$ & $2007-2009$ & $0.98 \pm 0.86$ & 3.2 \\
\hline (1915) Quetzalcoatl & 2.54 & 0.57 & 20.41 & $0.75 \pm 0.25$ & $0.09 \pm 0.09$ & $1953-2004$ & $0.97 \pm 1.10$ & 1.7 \\
\hline (3757) $1982 \mathrm{XB}$ & 1.83 & 0.45 & 3.87 & $0.50 \pm 0.10$ & $0.12 \pm 0.13$ & $1982-2008$ & $0.97 \pm 0.76$ & 2.7 \\
\hline (235756) $2004 \mathrm{VC}$ & 1.13 & 0.26 & 39.15 & $1.14 \pm 0.03$ & $0.04 \pm 0.01$ & $1992-2010$ & $0.96 \pm 0.79$ & 5.6 \\
\hline 2010 DH56 & 2.24 & 0.57 & 33.65 & $0.77 \pm 0.02$ & $0.02 \pm 0.01$ & 44 days & $0.95 \pm 0.70$ & 2.0 \\
\hline 2010 KY39 & 1.74 & 0.40 & 25.51 & $0.68 \pm 0.01$ & $0.03 \pm 0.00$ & 167 days & $0.94 \pm 0.85$ & 2.9 \\
\hline (162463) 2000 JH5 & 1.15 & 0.24 & 22.21 & $1.05 \pm 0.08$ & $0.16 \pm 0.04$ & $2000-2011$ & $0.94 \pm 0.75$ & 5.4 \\
\hline 2010 LG64 & 2.67 & 0.61 & 42.32 & $1.12 \pm 0.49$ & $0.01 \pm 0.00$ & 139 days & $0.94 \pm 2.30$ & 1.5 \\
\hline $2010 \mathrm{KZ} 117$ & 2.27 & 0.51 & 33.16 & $0.62 \pm 0.01$ & $0.09 \pm 0.02$ & 166 days & $0.93 \pm 0.80$ & 1.9 \\
\hline (177614) 2004 HK33 & 1.89 & 0.52 & 5.44 & $0.94 \pm 0.18$ & $0.19 \pm 0.14$ & 2001-2009 & $0.93 \pm 0.74$ & 2.5 \\
\hline (7335) $1989 \mathrm{JA}$ & 1.77 & 0.48 & 15.21 & $0.93 \pm 0.15$ & $0.32 \pm 0.15$ & $1989-2008$ & $0.93 \pm 0.91$ & 2.8 \\
\hline 2010 JL33 & 2.66 & 0.74 & 5.33 & $1.78 \pm 0.03$ & $0.05 \pm 0.01$ & $1997-2011$ & $0.93 \pm 0.71$ & 1.5 \\
\hline 2004 RA11 & 1.82 & 0.40 & 39.36 & $0.63 \pm 0.06$ & $0.14 \pm 0.04$ & $2004-2010$ & $0.92 \pm 0.78$ & 2.6 \\
\hline (175706) 1996 FG3 & 1.05 & 0.35 & 1.99 & $1.90 \pm 0.52$ & $0.03 \pm 0.03$ & $1996-2012$ & $0.92 \pm 0.73$ & 5.9 \\
\hline (68359) 2001 OZ13 & 1.52 & 0.17 & 9.86 & $0.62 \pm 0.15$ & $0.42 \pm 0.27$ & $1995-2010$ & $0.90 \pm 0.69$ & 3.4 \\
\hline (283729) 2002 UX & 1.47 & 0.16 & 20.21 & $0.65 \pm 0.13$ & $0.31 \pm 0.10$ & $2002-2012$ & $0.90 \pm 0.84$ & 3.5 \\
\hline (153814) 2001 WN5 & 1.71 & 0.47 & 1.92 & $0.93 \pm 0.01$ & $0.10 \pm 0.02$ & $1996-2010$ & $0.89 \pm 0.74$ & 2.8 \\
\hline (7822) $1991 \mathrm{CS}$ & 1.12 & 0.16 & 37.12 & $1.44 \pm 0.01$ & $0.09 \pm 0.01$ & $1991-2010$ & $0.89 \pm 0.66$ & 5.2 \\
\hline
\end{tabular}


Table 4-Continued

\begin{tabular}{|c|c|c|c|c|c|c|c|c|}
\hline NEA & $\begin{array}{c}a \\
(\mathrm{AU})\end{array}$ & $e$ & $\begin{array}{c}i \\
(\operatorname{deg})\end{array}$ & $\begin{array}{l}D \\
\quad(\mathrm{~km})\end{array}$ & $p_{V}$ & Arc & $\begin{array}{c}d a / d t \\
10^{-4} \mathrm{AU} / \mathrm{Myr}\end{array}$ & $\begin{array}{l}\Delta \rho \\
(\mathrm{km})\end{array}$ \\
\hline (142464) 2002 TC9 & 1.23 & 0.15 & 16.28 & $0.89 \pm 0.01$ & $0.12 \pm 0.02$ & $1991-2006$ & $0.88 \pm 0.73$ & 4.5 \\
\hline $2005 \mathrm{WE}$ & 1.15 & 0.25 & 12.36 & $1.15 \pm 0.01$ & $0.02 \pm 0.00$ & 2005-2011 & $0.88 \pm 0.68$ & 5.0 \\
\hline 2010 JF87 & 2.44 & 0.62 & 24.93 & $0.94 \pm 0.01$ & $0.04 \pm 0.01$ & 65 days & $0.86 \pm 0.66$ & 1.6 \\
\hline (222869) 2002 FB6 & 1.80 & 0.54 & 33.70 & $1.20 \pm 0.24$ & $0.14 \pm 0.03$ & 2002-2009 & $0.85 \pm 0.72$ & 2.5 \\
\hline 1989 AZ & 1.65 & 0.47 & 11.78 & $1.09 \pm 0.20$ & $0.03 \pm 0.02$ & $1989-2008$ & $0.85 \pm 0.69$ & 2.8 \\
\hline (90367) 2003 LC5 & 1.16 & 0.43 & 16.88 & $1.75 \pm 0.00$ & $0.05 \pm 0.01$ & $1983-2008$ & $0.85 \pm 0.57$ & 4.8 \\
\hline $2010 \mathrm{KK} 127$ & 2.23 & 0.42 & 6.94 & $0.50 \pm 0.02$ & $0.03 \pm 0.01$ & 177 days & $0.84 \pm 0.65$ & 1.8 \\
\hline (5143) Heracles & 1.83 & 0.77 & 9.03 & $4.84 \pm 0.38$ & $0.23 \pm 0.05$ & $1953-2011$ & $0.83 \pm 0.48$ & 2.3 \\
\hline (242187) 2003 KR18 & 2.34 & 0.48 & 5.58 & $0.65 \pm 0.13$ & $0.29 \pm 0.15$ & $1985-2010$ & $0.82 \pm 1.64$ & 1.6 \\
\hline (311321) 2005 NP1 & 1.83 & 0.30 & 34.70 & $0.58 \pm 0.11$ & $0.17 \pm 0.09$ & 2005-2012 & $0.82 \pm 0.70$ & 2.3 \\
\hline 2010 HD33 & 2.62 & 0.52 & 24.48 & $0.56 \pm 0.10$ & $0.27 \pm 0.13$ & 134 days & $0.80 \pm 0.63$ & 1.3 \\
\hline (10115) 1992 SK & 1.25 & 0.32 & 15.32 & $1.00 \pm 0.08$ & $0.28 \pm 0.14$ & 1953-1999 & $0.80 \pm 0.68$ & 4.0 \\
\hline (185716) 1998 SF35 & 1.69 & 0.27 & 35.19 & $0.63 \pm 0.10$ & $0.34 \pm 0.15$ & 1998-2009 & $0.80 \pm 0.62$ & 2.6 \\
\hline 2007 RM133 & 2.21 & 0.44 & 10.75 & $0.59 \pm 0.08$ & $0.27 \pm 0.08$ & $2007-2010$ & $0.80 \pm 0.66$ & 1.7 \\
\hline (254419) 2004 VT60 & 2.08 & 0.43 & 43.53 & $0.63 \pm 0.02$ & $0.41 \pm 0.09$ & 2001-2010 & $0.79 \pm 0.62$ & 1.9 \\
\hline (7839) $1994 \mathrm{ND}$ & 2.16 & 0.52 & 27.18 & $0.78 \pm 0.01$ & $0.18 \pm 0.03$ & $1994-2010$ & $0.79 \pm 0.57$ & 1.7 \\
\hline 2003 WO7 & 2.15 & 0.43 & 7.67 & $0.68 \pm 0.16$ & $0.11 \pm 0.07$ & 2003-2009 & $0.79 \pm 0.77$ & 1.7 \\
\hline 2004 TB18 & 1.82 & 0.45 & 13.20 & $0.86 \pm 0.01$ & $0.20 \pm 0.04$ & 2004-2011 & $0.78 \pm 0.62$ & 2.2 \\
\hline (137062) $1998 \mathrm{WM}$ & 1.22 & 0.32 & 22.52 & $1.27 \pm 0.04$ & $0.28 \pm 0.07$ & $1987-2010$ & $0.77 \pm 0.53$ & 4.0 \\
\hline 2010 FX80 & 2.17 & 0.45 & 36.94 & $0.68 \pm 0.13$ & $0.02 \pm 0.01$ & 84 days & $0.77 \pm 0.73$ & 1.7 \\
\hline $2006 \mathrm{AD}$ & 1.05 & 0.49 & 54.98 & $3.06 \pm 0.61$ & $0.04 \pm 0.02$ & 2006-2009 & $0.75 \pm 0.53$ & 4.9 \\
\hline 2010 GY6 & 1.29 & 0.23 & 21.91 & $1.10 \pm 0.08$ & $0.03 \pm 0.01$ & 240 days & $0.74 \pm 0.68$ & 3.6 \\
\hline 2005 SC71 & 1.91 & 0.38 & 32.37 & $0.74 \pm 0.15$ & $0.15 \pm 0.11$ & 2005-2010 & $0.74 \pm 0.71$ & 2.0 \\
\hline $2002 \mathrm{HF} 8$ & 2.32 & 0.49 & 4.78 & $0.71 \pm 0.16$ & $0.18 \pm 0.14$ & $2002-2009$ & $0.73 \pm 0.66$ & 1.5 \\
\hline $2009 \mathrm{WN}$ & 1.38 & 0.22 & 32.79 & $0.95 \pm 0.04$ & $0.06 \pm 0.01$ & $2002-2010$ & $0.73 \pm 0.59$ & 3.1 \\
\hline (315508) 2008 AB31 & 1.60 & 0.32 & 30.02 & $0.82 \pm 0.05$ & $0.02 \pm 0.01$ & $2002-2012$ & $0.72 \pm 0.62$ & 2.5 \\
\hline (29075) $1950 \mathrm{DA}$ & 1.70 & 0.51 & 12.18 & $2.00 \pm 0.20$ & $0.07 \pm 0.02$ & $1950-2012$ & $0.71 \pm 0.54$ & 2.2 \\
\hline (89959) 2002 NT7 & 1.74 & 0.53 & 42.33 & $1.41 \pm 0.08$ & $0.22 \pm 0.05$ & $1954-2011$ & $0.71 \pm 0.51$ & 2.2 \\
\hline (142563) 2002 TR69 & 1.66 & 0.34 & 20.49 & $0.86 \pm 0.14$ & $0.38 \pm 0.17$ & $1997-2012$ & $0.70 \pm 0.56$ & 2.3 \\
\hline 2010 OK126 & 1.96 & 0.45 & 52.54 & $0.85 \pm 0.01$ & $0.01 \pm 0.00$ & 129 days & $0.70 \pm 0.61$ & 1.8 \\
\hline
\end{tabular}


Table 4-Continued

\begin{tabular}{|c|c|c|c|c|c|c|c|c|}
\hline NEA & $\begin{array}{c}a \\
(\mathrm{AU})\end{array}$ & $e$ & $\begin{array}{c}i \\
(\operatorname{deg})\end{array}$ & $\begin{array}{l}D \\
\qquad(\mathrm{~km})\end{array}$ & $p_{V}$ & Arc & $\begin{array}{c}d a / d t \\
10^{-4} \mathrm{AU} / \mathrm{Myr}\end{array}$ & $\begin{array}{l}\Delta \rho \\
(\mathrm{km})\end{array}$ \\
\hline (162903) 2001 JV2 & 1.30 & 0.24 & 47.49 & $1.09 \pm 0.01$ & $0.06 \pm 0.02$ & $2001-2010$ & $0.69 \pm 0.55$ & 3.3 \\
\hline 2003 UL12 & 3.29 & 0.70 & 19.73 & $1.08 \pm 0.02$ & $0.20 \pm 0.05$ & $1998-2010$ & $0.69 \pm 0.50$ & 0.8 \\
\hline (139345) 2001 KA67 & 1.80 & 0.70 & 22.37 & $3.10 \pm 0.14$ & $0.04 \pm 0.01$ & $2001-2010$ & $0.68 \pm 0.47$ & 2.0 \\
\hline (3908) Nyx & 1.93 & 0.46 & 2.18 & $1.00 \pm 0.15$ & $0.16 \pm 0.08$ & 1980-2009 & $0.67 \pm 0.54$ & 1.7 \\
\hline (2102) Tantalus & 1.29 & 0.30 & 64.01 & $1.81 \pm 0.22$ & $0.21 \pm 0.08$ & $1975-2008$ & $0.66 \pm 0.51$ & 3.2 \\
\hline 2010 EH43 & 1.28 & 0.04 & 37.55 & $0.99 \pm 0.02$ & $0.05 \pm 0.01$ & $2010-2010$ & $0.66 \pm 0.52$ & 3.2 \\
\hline (2100) Ra-Shalom & 0.83 & 0.44 & 15.76 & $2.30 \pm 0.20$ & $0.13 \pm 0.03$ & 1975-2009 & $0.66 \pm 0.47$ & 6.1 \\
\hline 2007 HX4 & 1.32 & 0.33 & 56.56 & $1.39 \pm 0.04$ & $0.07 \pm 0.01$ & $1998-2010$ & $0.65 \pm 0.52$ & 3.0 \\
\hline (212546) 2006 SV19 & 2.13 & 0.51 & 7.34 & $1.06 \pm 0.23$ & $0.13 \pm 0.11$ & 2003-2009 & $0.65 \pm 0.66$ & 1.5 \\
\hline (66272) 1999 JW6 & 1.51 & 0.14 & 51.32 & $0.82 \pm 0.01$ & $0.42 \pm 0.06$ & 1999-2007 & $0.64 \pm 0.58$ & 2.4 \\
\hline (243566) $1995 \mathrm{SA}$ & 2.46 & 0.64 & 20.06 & $1.46 \pm 0.18$ & $0.09 \pm 0.03$ & $1991-2010$ & $0.64 \pm 0.54$ & 1.2 \\
\hline (15745) 1991 PM5 & 1.72 & 0.25 & 14.42 & $0.80 \pm 0.20$ & $0.23 \pm 0.07$ & $1982-2007$ & $0.63 \pm 0.55$ & 2.0 \\
\hline (103067) 1999 XA143 & 1.84 & 0.58 & 38.54 & $1.28 \pm 0.03$ & $0.25 \pm 0.05$ & $1994-2010$ & $0.63 \pm 0.48$ & 1.8 \\
\hline (85628) $1998 \mathrm{KV} 2$ & 1.59 & 0.33 & 13.03 & $1.01 \pm 0.06$ & $0.30 \pm 0.08$ & 1998-2012 & $0.62 \pm 0.48$ & 2.2 \\
\hline (2101) Adonis & 1.87 & 0.76 & 1.33 & $5.73 \pm 0.39$ & $0.04 \pm 0.01$ & $1936-2007$ & $0.61 \pm 0.36$ & 1.7 \\
\hline (66251) 1999 GJ2 & 1.54 & 0.20 & 11.28 & $1.22 \pm 0.23$ & $0.19 \pm 0.09$ & $1984-2012$ & $0.61 \pm 0.50$ & 2.2 \\
\hline 2000 HD74 & 2.92 & 0.59 & 49.30 & $0.83 \pm 0.02$ & $0.16 \pm 0.03$ & $2000-2010$ & $0.60 \pm 0.54$ & 0.8 \\
\hline 2000 JA3 & 2.25 & 0.46 & 10.18 & $0.77 \pm 0.15$ & $0.10 \pm 0.06$ & $2000-2010$ & $0.60 \pm 0.47$ & 1.2 \\
\hline (4953) $1990 \mathrm{MU}$ & 1.62 & 0.66 & 24.39 & $2.26 \pm 0.50$ & $0.79 \pm 0.25$ & 1974-2009 & $0.60 \pm 0.44$ & 2.0 \\
\hline $2009 \mathrm{XC} 2$ & 2.64 & 0.58 & 25.77 & $0.97 \pm 0.01$ & $0.26 \pm 0.03$ & $1954-2010$ & $0.60 \pm 0.46$ & 1.0 \\
\hline (40263) 1999 FQ5 & 1.49 & 0.16 & 25.84 & $0.95 \pm 0.19$ & $0.18 \pm 0.08$ & 1994-2012 & $0.60 \pm 0.54$ & 2.3 \\
\hline (12711) Tukmit & 1.19 & 0.27 & 38.48 & $1.90 \pm 0.40$ & $0.19 \pm 0.06$ & 1991-2009 & $0.60 \pm 0.47$ & 3.3 \\
\hline (8566) $1996 \mathrm{EN}$ & 1.51 & 0.43 & 37.96 & $1.57 \pm 0.26$ & $0.22 \pm 0.11$ & 1996-2011 & $0.60 \pm 0.46$ & 2.3 \\
\hline (66008) $1998 \mathrm{QH} 2$ & 1.43 & 0.36 & 61.01 & $1.48 \pm 0.28$ & $0.32 \pm 0.17$ & 1996-2008 & $0.59 \pm 0.49$ & 2.4 \\
\hline (2212) Hephaistos & 2.16 & 0.84 & 11.69 & $5.54 \pm 0.04$ & $0.16 \pm 0.03$ & $1978-2011$ & $0.58 \pm 0.35$ & 1.3 \\
\hline (215757) 2004 FU64 & 1.84 & 0.37 & 24.88 & $0.91 \pm 0.18$ & $0.19 \pm 0.06$ & 1999-2009 & $0.58 \pm 0.47$ & 1.6 \\
\hline (7888) $1993 \mathrm{UC}$ & 2.43 & 0.66 & 26.08 & $2.75 \pm 0.60$ & $0.18 \pm 0.06$ & $1989-2008$ & $0.58 \pm 0.48$ & 1.1 \\
\hline (17182) $1999 \mathrm{VU}$ & 1.39 & 0.55 & 9.27 & $2.88 \pm 0.19$ & $0.03 \pm 0.01$ & $1977-2007$ & $0.58 \pm 0.39$ & 2.5 \\
\hline (138847) 2000 VE62 & 1.62 & 0.29 & 22.19 & $0.97 \pm 0.15$ & $0.40 \pm 0.15$ & 1983-2012 & $0.58 \pm 0.48$ & 2.0 \\
\hline (3554) Amun & 0.97 & 0.28 & 23.36 & $3.33 \pm 0.02$ & $0.08 \pm 0.01$ & $1986-2012$ & $0.57 \pm 0.39$ & 4.1 \\
\hline
\end{tabular}


Table 4-Continued

\begin{tabular}{|c|c|c|c|c|c|c|c|c|}
\hline NEA & $\begin{array}{c}a \\
(\mathrm{AU})\end{array}$ & $e$ & $\begin{array}{c}i \\
(\mathrm{deg})\end{array}$ & $\begin{array}{l}D \\
\qquad(\mathrm{~km})\end{array}$ & $p_{V}$ & Arc & $\begin{array}{c}d a / d t \\
10^{-4} \mathrm{AU} / \mathrm{Myr}\end{array}$ & $\begin{array}{l}\Delta \rho \\
(\mathrm{km})\end{array}$ \\
\hline (17188) 1999 WC2 & 2.22 & 0.64 & 29.41 & $1.82 \pm 0.23$ & $0.15 \pm 0.04$ & $1990-2011$ & $0.57 \pm 0.39$ & 1.2 \\
\hline (87311) 2000 QJ1 & 1.59 & 0.51 & 7.69 & $1.95 \pm 0.14$ & $0.14 \pm 0.04$ & $1982-2011$ & $0.57 \pm 0.45$ & 2.0 \\
\hline (218863) 2006 WO127 & 2.19 & 0.55 & 11.00 & $1.24 \pm 0.01$ & $0.39 \pm 0.09$ & $2002-2010$ & $0.57 \pm 0.45$ & 1.2 \\
\hline (1864) Daedalus & 1.46 & 0.61 & 22.20 & $2.72 \pm 0.11$ & $0.27 \pm 0.06$ & 1971-2006 & $0.57 \pm 0.40$ & 2.2 \\
\hline (138883) 2000 YL29 & 1.54 & 0.34 & 21.89 & $1.22 \pm 0.22$ & $0.25 \pm 0.12$ & $1984-2011$ & $0.56 \pm 0.50$ & 2.1 \\
\hline (138013) 2000 CN101 & 1.60 & 0.63 & 15.95 & $3.52 \pm 0.23$ & $0.17 \pm 0.05$ & $1984-2012$ & $0.55 \pm 0.41$ & 1.9 \\
\hline (100004) $1983 \mathrm{VA}$ & 2.60 & 0.70 & 16.29 & $2.70 \pm 0.10$ & $0.07 \pm 0.01$ & $1983-2005$ & $0.55 \pm 0.40$ & 0.9 \\
\hline $2000 \mathrm{JY} 8$ & 2.78 & 0.60 & 16.53 & $1.11 \pm 0.22$ & $0.32 \pm 0.09$ & 2000-2009 & $0.54 \pm 0.45$ & 0.8 \\
\hline (12538) $1998 \mathrm{OH}$ & 1.54 & 0.41 & 24.53 & $1.66 \pm 0.33$ & $0.23 \pm 0.12$ & $1991-2010$ & $0.54 \pm 0.47$ & 2.0 \\
\hline 2010 AG79 & 2.91 & 0.58 & 32.97 & $0.89 \pm 0.01$ & $0.02 \pm 0.00$ & 70 days & $0.53 \pm 0.43$ & 0.8 \\
\hline 2010 VY190 & 1.81 & 0.31 & 19.97 & $0.95 \pm 0.22$ & $0.04 \pm 0.02$ & $2010-2011$ & $0.52 \pm 0.43$ & 1.5 \\
\hline (11500) Tomaiyowit & 1.08 & 0.36 & 10.31 & $0.74 \pm 0.01$ & $0.14 \pm 0.02$ & $1989-2007$ & $0.52 \pm 0.36$ & 3.2 \\
\hline 2005 CR37 & 1.91 & 0.47 & 26.07 & $1.20 \pm 0.24$ & $0.03 \pm 0.02$ & $2005-2010$ & $0.51 \pm 0.38$ & 1.4 \\
\hline 2010 LQ33 & 2.27 & 0.46 & 24.60 & $0.87 \pm 0.01$ & $0.04 \pm 0.01$ & 164 days & $0.51 \pm 0.45$ & 1.0 \\
\hline (40329) $1999 \mathrm{ML}$ & 2.27 & 0.45 & 2.51 & $0.96 \pm 0.23$ & $0.16 \pm 0.16$ & 1999-2009 & $0.50 \pm 0.43$ & 1.0 \\
\hline 2009 UV18 & 3.17 & 0.63 & 8.34 & $1.00 \pm 0.00$ & $0.71 \pm 0.10$ & $2004-2010$ & $0.50 \pm 0.36$ & 0.6 \\
\hline (243147) 2007 TX18 & 2.14 & 0.42 & 7.37 & $0.91 \pm 0.01$ & $0.28 \pm 0.06$ & 1991-2012 & $0.50 \pm 0.43$ & 1.1 \\
\hline $2007 \mathrm{XC} 10$ & 1.62 & 0.23 & 47.94 & $1.05 \pm 0.20$ & $0.03 \pm 0.01$ & $2007-2011$ & $0.50 \pm 0.45$ & 1.7 \\
\hline (162181) 1999 LF6 & 1.41 & 0.28 & 18.94 & $0.86 \pm 0.16$ & $0.13 \pm 0.09$ & $1999-2010$ & $0.49 \pm 0.43$ & 2.1 \\
\hline (16834) 1997 WU22 & 1.47 & 0.44 & 15.99 & $1.50 \pm 0.30$ & $0.40 \pm 0.12$ & $1988-2012$ & $0.49 \pm 0.43$ & 1.9 \\
\hline (144901) 2004 WG1 & 1.64 & 0.52 & 13.06 & $2.24 \pm 0.03$ & $0.04 \pm 0.01$ & 2004-2012 & $0.48 \pm 0.34$ & 1.6 \\
\hline (68372) 2001 PM9 & 1.62 & 0.42 & 8.10 & $1.73 \pm 0.45$ & $0.02 \pm 0.02$ & 2001-2011 & $0.48 \pm 0.44$ & 1.6 \\
\hline (159402) 1999 AP10 & 2.38 & 0.58 & 7.63 & $1.20 \pm 0.30$ & $0.34 \pm 0.23$ & 1999-2009 & $0.48 \pm 0.44$ & 0.9 \\
\hline (2201) Oljato & 2.17 & 0.71 & 2.52 & $1.80 \pm 0.10$ & $0.43 \pm 0.03$ & 1931-2012 & $0.48 \pm 0.40$ & 1.0 \\
\hline (52760) 1998 ML14 & 2.41 & 0.62 & 2.43 & $1.00 \pm 0.05$ & $0.27 \pm 0.24$ & $1998-2003$ & $0.47 \pm 0.39$ & 0.9 \\
\hline (159399) 1998 UL1 & 1.53 & 0.21 & 41.97 & $1.24 \pm 0.25$ & $0.27 \pm 0.09$ & $1998-2011$ & $0.47 \pm 0.41$ & 1.7 \\
\hline (88188) 2000 XH44 & 2.01 & 0.39 & 11.37 & $1.37 \pm 0.26$ & $0.37 \pm 0.17$ & $1991-2011$ & $0.46 \pm 0.37$ & 1.1 \\
\hline $2010 \mathrm{KH}$ & 2.76 & 0.55 & 14.57 & $1.03 \pm 0.02$ & $0.03 \pm 0.01$ & 222 days & $0.45 \pm 0.41$ & 0.7 \\
\hline (3103) Eger & 1.40 & 0.35 & 20.93 & $1.80 \pm 0.30$ & $0.39 \pm 0.12$ & $1982-2012$ & $0.45 \pm 0.41$ & 1.9 \\
\hline (85713) 1998 SS49 & 1.92 & 0.64 & 10.76 & $3.48 \pm 0.79$ & $0.08 \pm 0.04$ & $1998-2011$ & $0.44 \pm 0.37$ & 1.2 \\
\hline
\end{tabular}


Table 4-Continued

\begin{tabular}{|c|c|c|c|c|c|c|c|c|}
\hline NEA & $\begin{array}{c}a \\
(\mathrm{AU})\end{array}$ & $e$ & $\begin{array}{c}i \\
(\operatorname{deg})\end{array}$ & $\begin{array}{l}D \\
\qquad(\mathrm{~km})\end{array}$ & $p_{V}$ & Arc & $\begin{array}{c}d a / d t \\
10^{-4} \mathrm{AU} / \mathrm{Myr}\end{array}$ & $\begin{array}{c}\Delta \rho \\
(\mathrm{km})\end{array}$ \\
\hline (232382) 2003 BT47 & 2.34 & 0.49 & 7.49 & $1.12 \pm 0.00$ & $0.16 \pm 0.02$ & $2003-2011$ & $0.44 \pm 0.38$ & 0.9 \\
\hline 2010 AB78 & 2.25 & 0.55 & 33.24 & $1.52 \pm 0.01$ & $0.04 \pm 0.00$ & $2003-2010$ & $0.43 \pm 0.35$ & 0.9 \\
\hline (155334) 2006 DZ169 & 2.03 & 0.41 & 6.62 & $1.15 \pm 0.32$ & $0.20 \pm 0.18$ & $1983-2011$ & $0.43 \pm 0.36$ & 1.0 \\
\hline $2002 \mathrm{LV}$ & 2.32 & 0.60 & 29.53 & $1.73 \pm 0.35$ & $0.15 \pm 0.05$ & $2002-2012$ & $0.43 \pm 0.38$ & 0.9 \\
\hline (7350) $1993 \mathrm{VA}$ & 1.36 & 0.39 & 7.26 & $2.36 \pm 0.13$ & $0.05 \pm 0.01$ & $1986-2008$ & $0.43 \pm 0.36$ & 1.9 \\
\hline (54401) $2000 \mathrm{LM}$ & 1.71 & 0.26 & 18.95 & $1.19 \pm 0.19$ & $0.18 \pm 0.07$ & 1989-2009 & $0.42 \pm 0.38$ & 1.3 \\
\hline (54686) 2001 DU8 & 1.78 & 0.34 & 33.21 & $1.25 \pm 0.25$ & $0.34 \pm 0.09$ & $1988-2011$ & $0.42 \pm 0.33$ & 1.3 \\
\hline (85839) 1998 YO4 & 1.65 & 0.25 & 9.33 & $1.14 \pm 0.01$ & $0.41 \pm 0.08$ & $1993-2012$ & $0.42 \pm 0.34$ & 1.4 \\
\hline 2010 MU111 & 2.40 & 0.61 & 41.36 & $1.95 \pm 0.01$ & $0.02 \pm 0.00$ & 167 days & $0.42 \pm 0.36$ & 0.8 \\
\hline $2010 \mathrm{CM}$ & 2.62 & 0.54 & 7.42 & $1.17 \pm 0.23$ & $0.06 \pm 0.01$ & $2002-2010$ & $0.42 \pm 0.39$ & 0.7 \\
\hline (86067) 1999 RM28 & 1.82 & 0.32 & 30.54 & $1.25 \pm 0.21$ & $0.31 \pm 0.13$ & $1989-2011$ & $0.42 \pm 0.35$ & 1.2 \\
\hline 2000 WC67 & 2.69 & 0.57 & 10.00 & $1.31 \pm 0.02$ & $0.04 \pm 0.01$ & $2000-2010$ & $0.42 \pm 0.36$ & 0.7 \\
\hline (159608) $2002 \mathrm{AC} 2$ & 1.67 & 0.35 & 58.88 & $1.51 \pm 0.30$ & $0.20 \pm 0.06$ & $2002-2011$ & $0.42 \pm 0.36$ & 1.3 \\
\hline (230118) 2001 DB3 & 2.69 & 0.56 & 24.48 & $1.18 \pm 0.00$ & $0.15 \pm 0.02$ & $2001-2010$ & $0.42 \pm 0.30$ & 0.7 \\
\hline 2005 LY19 & 1.60 & 0.24 & 30.00 & $1.36 \pm 0.27$ & $0.26 \pm 0.08$ & $2005-2011$ & $0.41 \pm 0.35$ & 1.4 \\
\hline $2009 \mathrm{KC} 3$ & 3.21 & 0.70 & 10.01 & $2.19 \pm 0.45$ & $0.02 \pm 0.02$ & $2009-2010$ & $0.40 \pm 0.33$ & 0.5 \\
\hline 2010 NW1 & 3.39 & 0.62 & 39.55 & $1.16 \pm 0.23$ & $0.19 \pm 0.10$ & 109 days & $0.40 \pm 0.40$ & 0.4 \\
\hline (230979) 2005 AT42 & 2.86 & 0.61 & 11.23 & $1.52 \pm 0.04$ & $0.21 \pm 0.05$ & $2004-2010$ & $0.39 \pm 0.32$ & 0.6 \\
\hline (86326) 1999 WK13 & 1.84 & 0.36 & 34.30 & $1.35 \pm 0.03$ & $0.10 \pm 0.02$ & $1979-2011$ & $0.38 \pm 0.29$ & 1.1 \\
\hline 2004 YR32 & 3.06 & 0.70 & 20.52 & $2.29 \pm 0.28$ & $0.03 \pm 0.01$ & 2004-2010 & $0.38 \pm 0.29$ & 0.5 \\
\hline (153219) 2000 YM29 & 2.09 & 0.44 & 40.33 & $1.29 \pm 0.01$ & $0.05 \pm 0.01$ & $2000-2010$ & $0.38 \pm 0.33$ & 0.9 \\
\hline (1943) Anteros & 1.43 & 0.26 & 8.71 & $2.40 \pm 0.30$ & $0.15 \pm 0.05$ & $1973-2012$ & $0.38 \pm 0.34$ & 1.5 \\
\hline (12923) Zephyr & 1.96 & 0.49 & 5.29 & $2.06 \pm 0.01$ & $0.20 \pm 0.03$ & $1955-2012$ & $0.36 \pm 0.32$ & 0.9 \\
\hline (152558) $1990 \mathrm{SA}$ & 2.01 & 0.44 & 38.12 & $1.46 \pm 0.01$ & $0.16 \pm 0.02$ & $1990-2007$ & $0.36 \pm 0.29$ & 0.9 \\
\hline 2002 XG4 & 2.26 & 0.48 & 21.03 & $1.42 \pm 0.04$ & $0.05 \pm 0.01$ & $2002-2010$ & $0.36 \pm 0.26$ & 0.7 \\
\hline 2010 NG3 & 2.61 & 0.56 & 26.97 & $1.52 \pm 0.04$ & $0.10 \pm 0.02$ & 186 days & $0.35 \pm 0.29$ & 0.6 \\
\hline 2010 АН30 & 2.29 & 0.55 & 43.27 & $1.86 \pm 0.15$ & $0.02 \pm 0.00$ & 59 days & $0.35 \pm 0.27$ & 0.7 \\
\hline (214088) 2004 JN13 & 2.87 & 0.70 & 13.33 & $2.97 \pm 0.64$ & $0.25 \pm 0.08$ & $1975-2009$ & $0.35 \pm 0.26$ & 0.5 \\
\hline (68350) $2001 \mathrm{MK} 3$ & 1.67 & 0.25 & 29.56 & $1.79 \pm 0.33$ & $0.22 \pm 0.11$ & $1955-2007$ & $0.34 \pm 0.32$ & 1.1 \\
\hline 2003 UN12 & 2.16 & 0.40 & 6.91 & $1.25 \pm 0.25$ & $0.05 \pm 0.02$ & 2003-2012 & $0.34 \pm 0.25$ & 0.8 \\
\hline
\end{tabular}


Table 4-Continued

\begin{tabular}{|c|c|c|c|c|c|c|c|c|}
\hline NEA & $\begin{array}{c}a \\
(\mathrm{AU})\end{array}$ & $e$ & $\begin{array}{c}i \\
(\operatorname{deg})\end{array}$ & $\begin{array}{l}D \\
\qquad(\mathrm{~km})\end{array}$ & $p_{V}$ & Arc & $\begin{array}{c}d a / d t \\
10^{-4} \mathrm{AU} / \mathrm{Myr}\end{array}$ & $\begin{array}{c}\Delta \rho \\
(\mathrm{km})\end{array}$ \\
\hline (8201) 1994 AH2 & 2.54 & 0.71 & 9.56 & $1.86 \pm 0.18$ & $0.15 \pm 0.04$ & $1981-2010$ & $0.34 \pm 0.27$ & 0.6 \\
\hline (85818) 1998 XM4 & 1.66 & 0.42 & 62.72 & $2.22 \pm 0.02$ & $0.21 \pm 0.03$ & $1993-2012$ & $0.33 \pm 0.29$ & 1.1 \\
\hline (27346) $2000 \mathrm{DN} 8$ & 1.87 & 0.40 & 36.95 & $1.75 \pm 0.10$ & $0.26 \pm 0.04$ & $1978-2011$ & $0.33 \pm 0.29$ & 0.9 \\
\hline (247156) 2000 YH29 & 2.22 & 0.53 & 21.84 & $1.97 \pm 0.21$ & $0.03 \pm 0.01$ & $2000-2010$ & $0.32 \pm 0.27$ & 0.7 \\
\hline (153271) 2001 CL42 & 1.56 & 0.40 & 21.65 & $2.44 \pm 0.17$ & $0.04 \pm 0.01$ & $2001-2011$ & $0.32 \pm 0.22$ & 1.1 \\
\hline (241596) 1998 XM2 & 1.80 & 0.34 & 27.10 & $1.56 \pm 0.02$ & $0.10 \pm 0.02$ & $1952-2011$ & $0.31 \pm 0.23$ & 0.9 \\
\hline $2006 \mathrm{JT}$ & 2.40 & 0.48 & 36.42 & $1.52 \pm 0.32$ & $0.02 \pm 0.01$ & $2006-2010$ & $0.31 \pm 0.28$ & 0.6 \\
\hline (85804) 1998 WQ5 & 1.72 & 0.35 & 27.66 & $2.37 \pm 0.44$ & $0.24 \pm 0.09$ & $1989-2011$ & $0.30 \pm 0.24$ & 0.9 \\
\hline (5653) Camarillo & 1.79 & 0.30 & 6.87 & $1.54 \pm 0.02$ & $0.27 \pm 0.06$ & $1974-2012$ & $0.30 \pm 0.23$ & 0.9 \\
\hline 2010 LO97 & 2.58 & 0.53 & 21.66 & $1.63 \pm 0.31$ & $0.02 \pm 0.01$ & 182 days & $0.29 \pm 0.26$ & 0.5 \\
\hline 2009 WF104 & 3.07 & 0.66 & 17.00 & $2.23 \pm 0.03$ & $0.05 \pm 0.01$ & 2009-2010 & $0.29 \pm 0.22$ & 0.4 \\
\hline (153249) 2001 BW15 & 2.12 & 0.59 & 41.21 & $3.16 \pm 0.73$ & $0.18 \pm 0.11$ & $1989-2010$ & $0.29 \pm 0.21$ & 0.7 \\
\hline (154029) 2002 CY46 & 1.89 & 0.46 & 44.16 & $2.23 \pm 0.05$ & $0.10 \pm 0.02$ & $2002-2010$ & $0.29 \pm 0.24$ & 0.8 \\
\hline (159518) $2001 \mathrm{FF} 7$ & 2.10 & 0.44 & 47.51 & $1.78 \pm 0.03$ & $0.04 \pm 0.01$ & $2001-2010$ & $0.28 \pm 0.23$ & 0.6 \\
\hline (52387) 1993 OM7 & 1.28 & 0.19 & 24.15 & $1.22 \pm 0.25$ & $0.09 \pm 0.03$ & 1993-2009 & $0.28 \pm 0.24$ & 1.4 \\
\hline (153842) 2001 XT30 & 2.74 & 0.57 & 9.07 & $1.76 \pm 0.11$ & $0.19 \pm 0.04$ & $2000-2011$ & $0.28 \pm 0.24$ & 0.4 \\
\hline (217807) 2000 XK44 & 1.72 & 0.39 & 11.24 & $0.73 \pm 0.14$ & $0.28 \pm 0.18$ & $1975-2009$ & $0.28 \pm 0.25$ & 0.9 \\
\hline (162038) $1996 \mathrm{DH}$ & 1.59 & 0.28 & 17.23 & $1.96 \pm 0.22$ & $0.11 \pm 0.03$ & $1996-2012$ & $0.28 \pm 0.20$ & 1.0 \\
\hline (85709) 1998 SG36 & 1.65 & 0.34 & 24.84 & $2.23 \pm 0.14$ & $0.14 \pm 0.03$ & $1998-2012$ & $0.27 \pm 0.24$ & 0.9 \\
\hline $2005 \mathrm{QL}$ & 2.45 & 0.50 & 10.71 & $1.85 \pm 0.44$ & $0.02 \pm 0.01$ & $2005-2010$ & $0.27 \pm 0.25$ & 0.5 \\
\hline 2010 EH20 & 2.62 & 0.52 & 23.89 & $1.80 \pm 0.39$ & $0.03 \pm 0.03$ & $2010-2010$ & $0.27 \pm 0.24$ & 0.4 \\
\hline (36183) 1999 TX16 & 1.55 & 0.33 & 38.22 & $2.30 \pm 0.11$ & $0.09 \pm 0.02$ & $1997-2008$ & $0.26 \pm 0.21$ & 1.0 \\
\hline (11066) Sigurd & 1.39 & 0.38 & 36.89 & $2.78 \pm 0.12$ & $0.19 \pm 0.03$ & $1992-2012$ & $0.26 \pm 0.23$ & 1.1 \\
\hline (1620) Geographos & 1.25 & 0.34 & 13.34 & $3.90 \pm 0.40$ & $0.19 \pm 0.02$ & $1951-2012$ & $0.26 \pm 0.22$ & 1.3 \\
\hline (4183) Cuno & 1.98 & 0.63 & 6.70 & $5.62 \pm 0.46$ & $0.10 \pm 0.02$ & 1986-2012 & $0.26 \pm 0.20$ & 0.7 \\
\hline (3199) Nefertiti & 1.57 & 0.28 & 32.97 & $3.10 \pm 0.90$ & $0.10 \pm 0.10$ & $1982-2011$ & $0.26 \pm 0.24$ & 0.9 \\
\hline (5620) Jasonwheeler & 2.16 & 0.42 & 7.86 & $1.80 \pm 0.50$ & $0.09 \pm 0.09$ & $1955-2011$ & $0.25 \pm 0.21$ & 0.6 \\
\hline (304153) 2006 OU10 & 1.75 & 0.35 & 33.70 & $2.05 \pm 0.02$ & $0.03 \pm 0.01$ & 2006-2011 & $0.25 \pm 0.19$ & 0.8 \\
\hline (108519) $2001 \mathrm{LF}$ & 1.60 & 0.27 & 16.39 & $2.31 \pm 0.46$ & $0.02 \pm 0.01$ & $1989-2011$ & $0.25 \pm 0.20$ & 0.9 \\
\hline (234061) 1999 HE1 & 2.36 & 0.57 & 8.17 & $2.90 \pm 0.02$ & $0.02 \pm 0.00$ & $1999-2010$ & $0.24 \pm 0.18$ & 0.5 \\
\hline
\end{tabular}


Table 4-Continued

\begin{tabular}{|c|c|c|c|c|c|c|c|c|}
\hline NEA & $\begin{array}{c}a \\
(\mathrm{AU})\end{array}$ & $e$ & $\begin{array}{c}i \\
(\operatorname{deg})\end{array}$ & $\begin{array}{l}D \\
\qquad(\mathrm{~km})\end{array}$ & $p_{V}$ & Arc & $\begin{array}{c}d a / d t \\
10^{-4} \mathrm{AU} / \mathrm{Myr}\end{array}$ & $\begin{array}{l}\Delta \rho \\
(\mathrm{km})\end{array}$ \\
\hline $2004 \mathrm{~EB}$ & 3.13 & 0.66 & 21.36 & $2.54 \pm 0.24$ & $0.04 \pm 0.01$ & $1999-2010$ & $0.24 \pm 0.19$ & 0.3 \\
\hline $2001 \mathrm{RX11}$ & 2.77 & 0.54 & 13.05 & $1.82 \pm 0.04$ & $0.04 \pm 0.01$ & $2001-2010$ & $0.24 \pm 0.19$ & 0.4 \\
\hline (1863) Antinous & 2.26 & 0.61 & 18.40 & $3.23 \pm 0.60$ & $0.10 \pm 0.03$ & 1948-2009 & $0.24 \pm 0.22$ & 0.5 \\
\hline (5645) $1990 \mathrm{SP}$ & 1.35 & 0.39 & 13.51 & $1.67 \pm 0.02$ & $0.12 \pm 0.02$ & 1974-2009 & $0.24 \pm 0.18$ & 1.1 \\
\hline (285263) 1998 QE2 & 2.42 & 0.57 & 12.85 & $2.75 \pm 0.55$ & $0.06 \pm 0.02$ & 1998-2009 & $0.24 \pm 0.18$ & 0.4 \\
\hline (276049) 2002 CE26 & 2.23 & 0.56 & 47.31 & $3.33 \pm 0.95$ & $0.03 \pm 0.03$ & $2001-2010$ & $0.23 \pm 0.21$ & 0.5 \\
\hline 2010 LR68 & 3.03 & 0.61 & 4.58 & $2.25 \pm 0.15$ & $0.02 \pm 0.00$ & 2006-2011 & $0.23 \pm 0.21$ & 0.3 \\
\hline (4957) Brucemurray & 1.57 & 0.22 & 35.01 & $3.10 \pm 0.60$ & $0.17 \pm 0.06$ & $1976-2003$ & $0.23 \pm 0.20$ & 0.8 \\
\hline (138925) 2001 AU43 & 1.90 & 0.38 & 72.13 & $2.41 \pm 0.50$ & $0.11 \pm 0.03$ & 2001-2009 & $0.22 \pm 0.19$ & 0.6 \\
\hline (5731) Zeus & 2.26 & 0.65 & 11.43 & $5.23 \pm 0.69$ & $0.03 \pm 0.01$ & $1988-2006$ & $0.22 \pm 0.15$ & 0.5 \\
\hline (275611) 1999 XX262 & 1.53 & 0.18 & 8.23 & $2.43 \pm 0.18$ & $0.02 \pm 0.01$ & $1974-2010$ & $0.22 \pm 0.20$ & 0.8 \\
\hline (9950) ESA & 2.44 & 0.53 & 14.59 & $2.50 \pm 0.50$ & $0.10 \pm 0.03$ & 1990-2009 & $0.22 \pm 0.21$ & 0.4 \\
\hline (20460) Robwhiteley & 1.88 & 0.41 & 33.94 & $2.72 \pm 0.59$ & $0.20 \pm 0.14$ & 1954-2009 & $0.22 \pm 0.18$ & 0.6 \\
\hline (1685) Toro & 1.37 & 0.44 & 9.38 & $3.79 \pm 0.04$ & $0.25 \pm 0.04$ & $1948-2010$ & $0.21 \pm 0.18$ & 0.9 \\
\hline (248590) $2006 \mathrm{CS}$ & 2.91 & 0.70 & 52.30 & $4.73 \pm 0.84$ & $0.02 \pm 0.01$ & $1996-2011$ & $0.21 \pm 0.18$ & 0.3 \\
\hline (6455) $1992 \mathrm{HE}$ & 2.24 & 0.57 & 37.36 & $4.63 \pm 0.41$ & $0.23 \pm 0.05$ & 1989-2012 & $0.21 \pm 0.17$ & 0.4 \\
\hline (3122) Florence & 1.77 & 0.42 & 22.16 & $4.40 \pm 0.03$ & $0.23 \pm 0.05$ & 1979-2012 & $0.20 \pm 0.16$ & 0.6 \\
\hline (162998) 2001 SK162 & 1.93 & 0.47 & 1.68 & $0.87 \pm 0.01$ & $0.16 \pm 0.03$ & 1993-2009 & $0.20 \pm 0.16$ & 0.5 \\
\hline (248926) 2006 WZ2 & 1.69 & 0.33 & 24.66 & $2.91 \pm 0.23$ & $0.04 \pm 0.01$ & $1998-2010$ & $0.20 \pm 0.18$ & 0.6 \\
\hline (11398) 1998 YP11 & 1.72 & 0.39 & 15.02 & $1.32 \pm 0.35$ & $0.32 \pm 0.10$ & 1983-2012 & $0.19 \pm 0.21$ & 0.6 \\
\hline (4486) Mithra & 2.20 & 0.66 & 3.04 & $1.85 \pm 0.02$ & $0.30 \pm 0.06$ & $1987-2011$ & $0.19 \pm 0.17$ & 0.4 \\
\hline (6178) 1986 DA & 2.82 & 0.58 & 4.31 & $3.20 \pm 0.21$ & $0.16 \pm 0.03$ & $1977-2010$ & $0.19 \pm 0.17$ & 0.3 \\
\hline (248083) 2004 QU24 & 3.32 & 0.61 & 23.34 & $2.36 \pm 0.51$ & $0.13 \pm 0.07$ & 1993-2011 & $0.19 \pm 0.16$ & 0.2 \\
\hline (5626) $1991 \mathrm{FE}$ & 2.20 & 0.45 & 3.85 & $3.96 \pm 1.22$ & $0.15 \pm 0.15$ & $1970-2011$ & $0.19 \pm 0.19$ & 0.4 \\
\hline (100085) 1992 UY4 & 2.64 & 0.63 & 2.80 & $2.60 \pm 0.70$ & $0.02 \pm 0.02$ & 1979-2006 & $0.19 \pm 0.17$ & 0.3 \\
\hline (1866) Sisyphus & 1.89 & 0.54 & 41.19 & $6.60 \pm 0.19$ & $0.26 \pm 0.05$ & 1955-2012 & $0.18 \pm 0.12$ & 0.5 \\
\hline $2001 \mathrm{RC} 12$ & 3.22 & 0.64 & 27.33 & $3.20 \pm 0.40$ & $0.08 \pm 0.02$ & 2001-2007 & $0.17 \pm 0.16$ & 0.2 \\
\hline 2009 XE11 & 3.31 & 0.61 & 14.07 & $2.72 \pm 0.02$ & $0.04 \pm 0.01$ & $1997-2010$ & $0.17 \pm 0.16$ & 0.2 \\
\hline (4015) Wilson-Harri & 2.64 & 0.62 & 2.78 & $3.82 \pm 0.03$ & $0.05 \pm 0.01$ & $1949-2011$ & $0.17 \pm 0.13$ & 0.3 \\
\hline (152679) 1998 KU2 & 2.25 & 0.55 & 4.92 & $4.69 \pm 1.18$ & $0.02 \pm 0.01$ & $1998-2010$ & $0.16 \pm 0.24$ & 0.3 \\
\hline
\end{tabular}


Table 4-Continued

\begin{tabular}{|c|c|c|c|c|c|c|c|c|}
\hline NEA & $\begin{array}{c}a \\
(\mathrm{AU})\end{array}$ & $e$ & $\begin{array}{c}i \\
(\operatorname{deg})\end{array}$ & $\begin{array}{l}D \\
\quad(\mathrm{~km})\end{array}$ & $p_{V}$ & Arc & $\begin{array}{c}d a / d t \\
10^{-4} \mathrm{AU} / \mathrm{Myr}\end{array}$ & $\begin{array}{c}\Delta \rho \\
(\mathrm{km})\end{array}$ \\
\hline 2009 WO6 & 3.09 & 0.58 & 28.76 & $2.49 \pm 0.01$ & $0.03 \pm 0.01$ & 121 days & $0.16 \pm 0.14$ & 0.2 \\
\hline 2010 LF86 & 2.41 & 0.46 & 13.54 & $2.51 \pm 0.20$ & $0.03 \pm 0.01$ & $2010-2011$ & $0.16 \pm 0.13$ & 0.3 \\
\hline (5646) $1990 \mathrm{TR}$ & 2.14 & 0.44 & 7.91 & $2.72 \pm 0.53$ & $0.45 \pm 0.19$ & $1990-2006$ & $0.16 \pm 0.13$ & 0.3 \\
\hline (163691) 2003 BB43 & 2.41 & 0.52 & 40.89 & $3.45 \pm 0.54$ & $0.02 \pm 0.01$ & $1988-2007$ & $0.15 \pm 0.13$ & 0.3 \\
\hline (2368) Beltrovata & 2.10 & 0.41 & 5.24 & $3.00 \pm 0.49$ & $0.16 \pm 0.08$ & $1977-2012$ & $0.14 \pm 0.13$ & 0.3 \\
\hline (4055) Magellan & 1.82 & 0.33 & 23.24 & $2.78 \pm 0.15$ & $0.33 \pm 0.07$ & $1985-2012$ & $0.14 \pm 0.10$ & 0.4 \\
\hline (96189) Pygmalion & 1.82 & 0.31 & 13.99 & $3.61 \pm 0.16$ & $0.04 \pm 0.01$ & $1986-2011$ & $0.13 \pm 0.10$ & 0.4 \\
\hline (162566) 2000 RJ34 & 2.63 & 0.57 & 13.85 & $4.33 \pm 0.10$ & $0.07 \pm 0.01$ & $2000-2010$ & $0.13 \pm 0.09$ & 0.2 \\
\hline (6050) Miwablock & 2.20 & 0.44 & 6.40 & $3.54 \pm 0.88$ & $0.19 \pm 0.15$ & $1953-2012$ & $0.12 \pm 0.11$ & 0.3 \\
\hline (5587) $1990 \mathrm{SB}$ & 2.39 & 0.55 & 18.10 & $4.86 \pm 0.85$ & $0.25 \pm 0.11$ & $1953-2012$ & $0.12 \pm 0.11$ & 0.2 \\
\hline (3752) Camillo & 1.41 & 0.30 & 55.55 & $2.31 \pm 0.09$ & $0.21 \pm 0.04$ & $1976-2003$ & $0.12 \pm 0.11$ & 0.5 \\
\hline (20826) 2000 UV13 & 2.42 & 0.63 & 31.87 & $5.10 \pm 1.00$ & $0.27 \pm 0.09$ & $1953-2012$ & $0.11 \pm 0.10$ & 0.2 \\
\hline (54789) $2001 \mathrm{MZ7}$ & 1.78 & 0.29 & 24.46 & $1.57 \pm 0.02$ & $0.86 \pm 0.14$ & $1978-2011$ & $0.11 \pm 0.10$ & 0.3 \\
\hline (52762) 1998 МТ24 & 2.42 & 0.65 & 33.88 & $6.74 \pm 0.19$ & $0.05 \pm 0.01$ & $1953-2008$ & $0.10 \pm 0.08$ & 0.2 \\
\hline (5370) Taranis & 3.33 & 0.63 & 19.09 & $5.33 \pm 0.08$ & $0.04 \pm 0.01$ & $1986-2011$ & $0.10 \pm 0.09$ & 0.1 \\
\hline (19764) 2000 NF5 & 2.23 & 0.44 & 1.33 & $1.57 \pm 0.07$ & $0.34 \pm 0.08$ & $1990-2012$ & $0.09 \pm 0.08$ & 0.2 \\
\hline (88263) $2001 \mathrm{KQ} 1$ & 2.10 & 0.43 & 38.83 & $5.71 \pm 0.03$ & $0.04 \pm 0.01$ & $1998-2008$ & $0.09 \pm 0.07$ & 0.2 \\
\hline (1580) Betulia & 2.20 & 0.49 & 52.11 & $5.39 \pm 0.54$ & $0.08 \pm 0.01$ & $1950-2010$ & $0.09 \pm 0.07$ & 0.2 \\
\hline (17274) $2000 \mathrm{LC} 16$ & 2.72 & 0.56 & 5.62 & $3.18 \pm 0.11$ & $0.04 \pm 0.01$ & $1955-2011$ & $0.08 \pm 0.07$ & 0.1 \\
\hline (1980) Tezcatlipoca & 1.71 & 0.36 & 26.86 & $5.99 \pm 0.08$ & $0.14 \pm 0.02$ & $1950-2012$ & $0.08 \pm 0.07$ & 0.3 \\
\hline (26760) $2001 \mathrm{KP} 41$ & 2.87 & 0.55 & 10.91 & $5.40 \pm 0.37$ & $0.04 \pm 0.01$ & $1996-2011$ & $0.08 \pm 0.06$ & 0.1 \\
\hline (25916) $2001 \mathrm{CP} 44$ & 2.56 & 0.50 & 15.75 & $5.68 \pm 0.03$ & $0.26 \pm 0.05$ & $1973-2012$ & $0.07 \pm 0.06$ & 0.1 \\
\hline (143651) 2003 QO104 & 2.13 & 0.53 & 11.62 & $2.29 \pm 0.54$ & $0.14 \pm 0.14$ & $1981-2012$ & $0.07 \pm 0.07$ & 0.2 \\
\hline (1627) Ivar & 1.86 & 0.40 & 8.45 & $8.37 \pm 0.08$ & $0.13 \pm 0.03$ & $1929-2012$ & $0.07 \pm 0.05$ & 0.2 \\
\hline (21088) 1992 BL2 & 1.71 & 0.24 & 38.46 & $4.23 \pm 0.11$ & $0.21 \pm 0.05$ & $1990-2012$ & $0.05 \pm 0.04$ & 0.2 \\
\hline (3691) Bede & 1.77 & 0.28 & 20.36 & $1.80 \pm 0.11$ & $0.59 \pm 0.12$ & $1975-2012$ & $0.04 \pm 0.03$ & 0.1 \\
\hline (887) Alinda & 2.48 & 0.57 & 9.36 & $4.79 \pm 0.23$ & $0.34 \pm 0.06$ & $1918-2008$ & $0.03 \pm 0.03$ & 0.1 \\
\hline (16064) Davidharvey & 2.85 & 0.59 & 4.54 & $4.11 \pm 0.59$ & $0.02 \pm 0.01$ & 1994-2009 & $0.02 \pm 0.02$ & 0.0 \\
\hline (433) Eros & 1.46 & 0.22 & 10.83 & $33.60 \pm 0.12$ & $0.25 \pm 0.06$ & $1893-2012$ & $0.02 \pm 0.01$ & 0.1 \\
\hline (1036) Ganymed & 2.66 & 0.53 & 26.70 & $36.75 \pm 0.31$ & $0.23 \pm 0.03$ & $1924-2012$ & $0.01 \pm 0.01$ & 0.0 \\
\hline
\end{tabular}


